\title{
Jóvenes Mexicanos
}

Recebido em

15/02/2011

\section{em Medio de la Crisis Económica:}

\author{
Los Problemas de la
}

Integración Laboral
Aprovado em 06/06/2011
Minor Mora Salas ${ }^{1}$
Orlandina de Oliveira ${ }^{2}$

Resumen El artículo analiza el impacto de la crisis financiera global sobre el mercado laboral mexicano, centrando su interés en el estudio de las formas de participación de la mano de obra más joven en el mercado de trabajo. En concreto se estudia cómo la crisis acentuó, de manera diferencial, la mayor vulnerabilidad laboral de las y los trabajadores adolescentes, al fomentar procesos de informalización, precarización y desprotección laboral más intensos. Adicionalmente, se observa que la crisis tuvo impactos diferenciales a nivel regional, siendo que las entidades federativas con mayor integración a la economía norteamericana -como las de norte del país- fueron las más afectadas por esta crisis, en contraste con aquellas que presentan un bajo nivel de integración a los procesos de globalización económica en general y a la economía de los EEUU en particular -como las de la región sur del país. Finalmente, se observa que esta crisis también afectó, principalmente, a las entidades federativas que tenían mercados laborales más modernos, es decir, aquellas en los que predominan tasas altas de asalarización de las relaciones laborales.

Palabras claves México, regiones, crisis, jóvenes, desempleo, informalidad, precariedad laboral, vulnerabilidad laboral.

Abstract The article analyzes the impact of the global financial crisis on the Mexican labor market, with a focus in the study of forms for participation the

${ }^{1}$ Profesor-investigador del Centro de Estudios Sociológicos de El Colegio de México.

2Profesorainvestigadora del Centro de Estudios Sociológicos de El Colegio de México. 
${ }^{3}$ No hay que olvidarse, asimismo, del impacto económico negativo generado por el temor de propagación de la gripe H1N1. En el caso mexicano, los efectos de la crisis global se sumaron a los provocados por el impacto de esta pandemia en algunos sectores de la economía nacional. workers young in the labor market. Specifically, it examines how the crisis deepened, so differential, the greater vulnerability of labor and young workers, to encourage processes of precarious and unprotected work more intense. Furthermore, we find that the crisis had differential impacts at the regional level, being that states with greater integration into the American economy, as the north of the country were most affected by this crisis, in contrast to those that have a low level of integration with the processes of economic globalization in general and the U.S. economy in particular, as those of the southern region. Finally, we note that this crisis also affected, mainly, to the states that had modern labor markets, in other words, those in the prevailing high rates of wage-earning labor relations.

Keys words Mexico, regions, crisis, youth unemployment, informality, labor insecurity, employment vulnerability.

\section{Introducción}

México experimentó, en el 2009, la más aguda contracción económica en su historia reciente. Como ha sido ampliamente documentado (CEPAL, 2009), la crisis mundial se transmitió a las economías latinoamericanos por diversos medios, entre los cuales cabe mencionar: la caída de las exportaciones debido a la reducción de la demanda externa, la disminución de las inversiones extranjeras directas, la menor disponibilidad de créditos internacionales, el descenso de las remesas y la contracción de los ingresos derivados del turismo internacional. La dependencia comercial mexicana respecto del mercado norteamericano potenció el impacto de estos factores, contribuyendo así a que la crisis impactará con mayor fuerza a nuestro país ${ }^{3}$.

Esta recesión, desencadenada por la crisis financiera global, trajo una caída abrupta del Producto Interno Bruto y un proceso importante de pauperización de los hogares mexicanos. En parte, el deterioro económico de las unidades familiares está asociado con el desempleo de alguno de sus integrantes. Uno de los sectores más afectados por este fenómeno es la población joven, cuyas tasas de desocupación superan, ampliamente, el promedio nacional. 
El interés en este texto es analizar la situación laboral de las y los jóvenes mexicanos en el contexto recesivo de finales de la primera década del presente siglo. Los efectos de una crisis económica -en el ámbito laboral- pueden manifestarse de diversos modos, como por ejemplo, el aumento de los niveles de desempleo o del subempleo, la expansión de las actividades informales, el deterioro de las condiciones laborales, la contracción de los salarios o el aumento de la inactividad. Se privilegia en este trabajo el incremento de los niveles de desempleo, la expansión de las actividades informales y la reducción de la protección laboral. Pero también hacemos mención, cuando se hace necesario, a las variaciones en las tasas de participación económica, subempleo, asalarización de la fuerza de trabajo e inactividad.

Tratamos de brindar respuesta a cuatro cuestiones. Primero, conocer sí el impacto de la crisis en el nivel nacional, de acuerdo con los indicadores seleccionados, ha sido más acentuado en la población joven ${ }^{4}$ en comparación con la adulta. Segundo examinar sí dicho impacto se manifiesta de modo diferenciado en ambos grupos etarios. Tercero, explorar cómo se expresan las consecuencias laborales de la crisis en diferentes mercados laborales; estos últimos son definidos en función del grado de asalarización de los mercados de trabajo estatales. Cuarto, identificar los contextos laborales que muestran las repercusiones más severas sobre la mano de obra joven.

El análisis se centra en las personas jóvenes de 14 a 19 años de edad y utiliza la información estadística proporcionada por el INEGI en la consulta interactiva de la Encuesta de Ocupación y Empleo (ENOE) del segundo semestre de 2008 y 2009, así como la obtenida mediante el procesamiento de las bases de datos de la ENOE para los mismos períodos. El trabajo se inicia con un breve recuento acerca de la presencia de la mano de obra joven en los mercados de trabajo desde fines del siglo pasado. En seguida se analizan las repercusiones de la crisis económica en la situación laboral de la mano de obra juvenil en el nivel nacional. Posteriormente, se destaca el impacto diferencial de la crisis en los contextos laborales más o menos asalariados. El texto termina con el señalamiento de los principales resultados y subrayando los desafíos que enfrenta el país en materia de integración laboral de la población joven.
${ }^{4}$ Incluimos en este grupo a la población entre 14 y 29 años. Mientras que en el grupo de población adulta se considera a quienes tienen 30 años y más. 
${ }^{5}$ Véase, entre otros, Quintana, 1996; Rendón y Salas, 1996; INEGI, 2000; Rendón y Salas, 2000; Navarrete López, 2001; Camarena, 2005, Meza Romero, 2006, Hernández Pérez, 2007.

${ }^{6}$ Para la construcción del índice recurrimos al análisis factorial y la aplicación de análisis de conglomerados que nos permitió agrupar el índice en las categorías que presentaban la menor variabilidad interna y las mayores diferencias entre grupos (véase, Oliveira, 2006). Los indicadores utilizados para medir el grado de precariedad fueron los siguientes: contar o no con local de trabajo, con alguna prestación laboral, con contrato permanente así como desempeñar actividades relacionados con los estudios, la duración de la jornada de trabajo y el nivel salarial.

${ }^{7}$ Tasa de condiciones críticas de ocupación se refiere al "porcentaje de la

\section{La precariedad del trabajo juvenil como un rasgo persistente}

El estudio de las repercusiones de la reciente crisis económica en la situación laboral de la mano de obra joven debe enmarcarse, como se mencionó anteriormente, en el contexto del cambio de modelo de desarrollo sustentado en la ejecución de las políticas de apertura comercial y liberalización económica.

En un período histórico caracterizado por la restructuración productiva, la desregulación laboral y la apertura comercial, la calidad de los empleos se ha deteriorado. La pérdida de importancia del empleo público, la precarización del trabajo asalariado y a la expansión del autoempleo de subsistencia explica, en parte, este proceso. El deterioro laboral acaecido en México, en los años de transición y consolidación del nuevo modelo económico, ha sido caracterizado como un rasgo estructural del nuevo modelo de acumulación de capital, el cual busca disminuir los costos de producción mediante el abaratamiento de la fuerza de trabajo con miras a lograr una mayor competitividad internacional (De la Garza, 1990; Hernández Laos y Velázquez, 2003; Hernández Laos y otros, 2000).

La evidencia empírica muestra que, en la historia reciente del país, gran parte de la mano de obra juvenil ha ocupado los puestos de trabajo más precarios, esto es, sin estabilidad, seguridad social, prestaciones laborales y remuneraciones deprimidas. Cuanto más precoz la inserción laboral de los hombres y mujeres jóvenes, mayor su vulnerabilidad laboral. Este patrón de absorción de la mano de obra juvenil es resultado de la falta de oportunidades laborales, la pérdida de calidad de los nuevos empleos, los bajos niveles de escolaridad, la menor experiencia laboral y el menor poder de negociación de quienes se integran precozmente al mundo del trabajo (Oliveira, 2006 y 2009; Mora y Oliveira 2009; Castillo, 2008).

Investigaciones realizadas a fines del siglo pasado ya mostraban la precaria inserción laboral de los y las jóvenes -asalariados y no asalariados de 12 a 24 años- definida de acuerdo con sus niveles de ingreso, jornada de trabajo y prestaciones laborales (Navarrete, 1998; Rendón y Salas, 1996). El panorama que proporciona diversos indicadores de la precariedad del empleo asalariado al inicio del siglo XXI tampoco es muy alentador. ${ }^{5}$ 
De acuerdo con un índice de calidad/precariedad del empleo asalariadoelaborado a partir de los datos de la Encuesta Nacional de Juventud, 2000 (ENJ, 2000)- 36.1\% de las y los jóvenes asalariados -de 12 a 29 años- presentaba una inserción laboral de alta o muy alta precariedad ${ }^{6}$. El análisis de las condiciones críticas de ocupación ${ }^{7}$ completa el panorama de la precariedad laboral de contingentes importantes de la población joven. Este indicador capta situaciones extremas que podrían ser definidas como hipercríticas, ya que considera como criterio de diferenciación el trabajo de tiempo completo y la remuneración inferior al salario mínimo -monto insuficiente para cubrir el costo de una canasta normativa de satisfactores esenciales-. ${ }^{8}$

Datos de 2004 muestran que las condiciones críticas de ocupación ya eran -desde entonces- más acentuadas en la población joven -12 a 29 años- comparativamente con el total de los trabajadores ocupados ( 31.3 frente a 26.1\%) (Oliveira, 2009). La situación de los varones asalariados de 12 a 17 años era aún más dramática: solamente 3 de cada 100 lograban acceder a un empleo con los estándares laborales mínimos (Mora Salas y Oliveira, 2009).

Los puestos de trabajo más precarios, en casi su totalidad, no tenían contrato laboral y las actividades realizadas no se relacionaban con los estudios de las y los jóvenes. Además, una gran parte de estos empleos no contaba con prestaciones laborales ni sueldo base, los salarios pagados eran inferiores a 1.5 salarios mínimos y en más de la mitad de los casos, exigía jornadas laborales excesivas.

Los empleos de precariedad extrema se han nutrido, por lo general, de fuerza de trabajo muy joven, se trata de menores de 20 años con baja escolaridad que, con frecuencia, desempeñan actividades manuales no calificadas, laboran en la agricultura, en los servicios distributivos (comercio y transporte) o en los servicios personales (Oliveira, 2006). Como se verá a lo largo de este trabajo, la situación de precariedad laboral de las personas jóvenes se agudiza en el marco de la crisis económica global a causa del aumento del desempleo, la informalidad y/o la ausencia de seguridad social en contextos laborales específicos. población ocupada, que se encuentra trabajando menos de 35 horas a la semana por razones de mercado, más la que trabaja más de 35 horas semanales con ingresos mensuales inferiores al salario mínimo, la que labora más de 48 horas semanales ganando hasta dos salarios mínimos" (INEGI). A partir de esta tasa construimos otra que incluye a los trabajadores sin remuneración (véase Oliveira, 2009).

${ }^{8}$ De acuerdo con los datos que proporcionaron Julio Bolvinik y Araceli Damián, en 2004 se requerían cerca de 1.5 salarios mínimos para adquirir una canasta normativa de satisfactores esenciales per capita. Véase, Bolvinik y Damián (2003) para una lista de los ítems incluidos en dicha canasta. 
`La población económicamente activa (PEA) incluye a los ocupados y los desocupados.

${ }^{10}$ Las cifras exactas son de 13,193.510 de jóvenes de 14 a 19 años en el país en 2009, de estos 3, 969,615 estaban en la PEA; 7 , 410,136 eran estudiantes, 1, 390,027 se dedicaban a labores del hogar, 49,771 estaban discapacitados y 373,961 ni estudiaban ni trabajaban en actividades domésticas y extra doméstica (ENOE, 2009).

"Sería de interés analizar las consecuencias de la crisis económica en la población joven que estudia, la que se dedica a la realización de los quehaceres del hogary la que no estudia ni está en la fuerza de trabajo.

${ }^{12}$ Tasa de participación se refiere al "porcentaje que representa la población económicamente activa (PEA) respecto a la población de 14 y más años de edad" (INEGI). La

\section{Impacto de la crisis econômica en el trabajo juvenil \\ en el nivel nacional}

En este texto se privilegia el análisis de los y las jóvenes de 14 a 19 años que participan en la población económicamente activa (PEA). En 2009, la cifra estimada de población total en este grupo de edad era poco más de 13 millones, de los cuales casi 4 millones formaban parte de la $\mathrm{PEA}^{9}$, esto es representaban $30.1 \%$ del total de la población joven. La contraparte, es decir 69.9\%, se encontraba fuera de la PEA, La mayoría de estos jóvenes inactivos se dedicaba a estudiar -en 2009 la cifra estimada de estudiante era de casi 7 millones y medio-. Cerca de 1 millón cuatrocientos mil jóvenes reportó no trabajar ni estudiar, sino estar vinculado a la realización de labores de la casa, grupo conformado sobre todo por mujeres. Otros más no estudiaban ni participaban en el mercado de trabajo ni en las actividades del hogar porque se encontraban discapacitados para hacerlo. Por último están los que desistieron de encontrar una actividad laboral, abandonaron los estudios y no participan en las actividades del hogar (ENOE, 2009). ${ }^{10}$

En este análisis profundizamos en el impacto de la crisis en la población joven activo económicamente por constituir, como hemos señalado, un grupo que ingresa al mercado de trabajo en condiciones muy adversas. Queda pendiente para futuros trabajos estudiar el impacto de la crisis en la población joven inactiva ${ }^{11}$. Por ahora, nos limitaremos a señalar el aumento de la inactividad juvenil en contextos laborales específicos cuando sea pertinente.

Inicialmente con el propósito que profundizar en el impacto diferencial de la crisis sobre la situación laboral de la fuerza de trabajo juvenil en comparación con los grupos de mayor edad, se utilizan dos tipos de indicadores: unos que permiten examinar el mayor o menor grado de utilización de la fuerza de trabajo -tasas de participación ${ }^{12}$, desempleo ${ }^{13}$ y subempleo ${ }^{14}$ - y otros que hacen posible observar el mayor o menor grado de precariedad de la situación laboral de los y las jóvenes -la asalarización ${ }^{15}$, la participación en el sector informal ${ }^{16}$ y en ocupaciones sin protección laboral ${ }^{17}$-. La exploración de este conjunto de indicadores nos posibilita sopesar en qué aspectos la situación laboral de la mano de obra joven ha sido afectada por la crisis económica que azotó a México desde finales del 2008. 
Para contar con una visión general y subrayar la diversidad de situaciones entre grupos edad, y entre la mano de obra juvenil masculina y femenina, se compara en un primer momento a los más jóvenes -14 a 19 años- con la población joven de mayor edad -20 a 29 años-y con los adultos -30 años y más-. Se empieza con el análisis del grado de utilización de la fuerza de trabajo y posteriormente se centra en los indicadores de precariedad de la situación laboral. Para captar posibles impactos de la recesión económica nos referimos tanto a los niveles de los indicadores como a las variaciones entre 2008-200918.

La participación económica suele ser menor en la población joven que en la adulta, y en las mujeres en comparación con los hombres. En el caso del desempleo ocurre lo contrario: es más elevada en personas jóvenes y en mujeres en comparación con adultos y hombres. ${ }^{19}$ Cifras para 2008 y 2009 reafirman estos resultados (cuadro 1).

En un contexto de desaceleración del crecimiento económico se podría esperar una reducción de las tasas de participación en el mercado de trabajo. Este efecto sería producto de la contracción del nivel de ocupación de la mano de obra frente a la destrucción de puestos de trabajo y/o la falta de creación de oportunidades de empleo. Pero, en otras situaciones de contracción económica se ha observado un incremento de las tasas de participación económica por parte de algunos sectores de la fuerza de trabajo. Esta aparente contradicción se explica por el aumento del desempleo o la expansión del autoempleo en un contexto de escasez de recursos económicos en el ámbito familiar (Oliveira, 1989, García y Oliveira, 1994; Cortés y Rubalcava, 1991).

En la coyuntura actual, al comparar datos nacionales agregados -correspondientes al segundo trimestre de 2008-2009- encontramos que la participación económica disminuye y el desempleo incrementa en todos los grupos de edad, con la excepción de la mujeres de 50 a 59 años cuya presencia en la actividad económica se mantiene sin grandes alteraciones (cuadro 2).

La población menor de 20 años fue la más afectada. En efecto, la reducción de su participación económica así como el incremento de sus niveles de desempleo han sido más acentuados. Asimismo, los hombres redujeron más su presencia en la actividad económica y enfrentaron mayores niveles de

tasa de participación de jóvenes de 14 a 19 años se refiere al porcentaje que representa la PEA de 14 a 19 años respecto a la población de 14 a 19 años.

${ }^{13}$ Tasa de desocupación o de desempleo se refiere al "porcentaje de la población económicamente activa (PEA) que se encuentra sin trabajar pero que está buscando trabajo" (INEGI).

${ }^{14}$ Tasa de subocupación o subempleo se refiere al "porcentaje de la población ocupada que tiene la necesidad y disponibilidad de ofertar más tiempo de trabajo de lo que su ocupación actual les permite" (INEGI)

${ }^{15}$ Tasa de trabajo asalariado o de asalarización se refiere al "porcentaje de la población ocupada que percibe de la unidad económica para la que trabaja un sueldo, salario o jornal, por las actividades realizadas “(INEGI). 
${ }^{16}$ Tasa de ocupación en el sector informal: "La población ocupada, que trabaja para una unidad económica que opera a partir de los recursos del hogar, pero sin constituirse como empresa, de modo que la actividad no tiene una situación identificable e independiente de ese hogar. La manera operativa de establecer esto es que la actividad no lleva una contabilidad bajo las convenciones que permiten que sea auditada". (INEGI).

${ }^{17}$ La ocupaciones no protegidas según la definición del INEGI incluye a la "población ocupada en el sector informal, trabajo doméstico remunerado, agricultura de auto subsistencia, trabajadores subordinados y remunerados que no cuentan con acceso a las instituciones de salud; así como trabajadores cuentas propias en actividades agropecuarias orientadas al mercado que no tienen acceso a las instituciones de salud, con respecto al total de la po- desempleo que las mujeres. A pesar de lo anterior, ellas siguen presentando tasas de desempleo superiores. En otras palabras, el incremento del desempleo de los varones no ha sido suficiente para contrarrestar las diferencias por sexo: en 2009 la tasa de desempleo en los hombres de 14 a 19 años fue de $9.7 \%$ frente a $10.4 \%$ de las mujeres en el mismo grupo de edad (cuadro 1)

Lo anterior contrasta con la evolución del subempleo. En este particular la situación de la fuerza laboral más joven resulta ser más favorable, ya que la mano de obra adulta, sobretodo la que se encuentra entre los 50 y 59 años, ha sido la más afectada. En otras palabras los trabajadores en etapas más avanzadas de su vida laboral se han enfrentado a la necesidad y disponibilidad de trabajar más horas sin lograrlo. Nótese que el grupo de 60 años y más, al igual que los muy jóvenes, ha sido menos afligido por el subempleo (cuadros 1 y 2 ).

En cuanto a la precariedad del trabajo -vista mediante la reducción del trabajo asalariado, informalidad y desprotección laboral- las cifras globales para el conjunto del país ocultan, en cierta medida, el acentuado deterioro laboral ocasionado por la recesión económica que se manifiesta en diferentes contextos laborales como se verá más adelante. En el nivel nacional, la tasa de asalarización ${ }^{20}$ de la mano de obra no presenta grandes variaciones por edad entre 2008-2009, excepción hecha para las mujeres de 60 años y más que donde se muestra un ligero incremento. Nótese que alrededor de 65\% de los y las jóvenes de 14 a 19 años desempeñaban trabajos asalariados, cifra que es inferior solamente a la mano de obra de 20 a 39 años de edad (cuadros 1 y 2). En otras palabras, la inserción laboral de la mano de obra joven es predominantemente como trabajadores subordinados.

En cuanto a la participación en el sector informal (negocios vinculados a los recursos del hogar según la definición del INEGI) las variaciones entre 2008-2009 son mínimas. Adviértase que encontramos una ligera expansión de las actividades informales -2.6\%- solamente en el caso de las mujeres muy jóvenes (cuadro 2). Es preciso tener en cuenta que en el total nacional, las diferencias entre grupos de edad -así como entre hombres y mujeres-en cuanto a los niveles de informalidad siguen siendo importantes. La presencia en el sector informal es mayor (32.5\%- cifra para 2009) en los hombres muy jóvenes (14 a 19 años) en comparación con los demás grupos de edad. 
En el caso de las jóvenes esto no ocurre debido a que las mujeres de 50 años y más las sobrepasan en este tipo de actividades. El panorama es distinto para los hombres y mujeres de 20 a 29 años, puede observarse que su presencia en las actividades informales es más reducida que en los demás grupos de edad (cuadro 1).

Las variaciones en la población ocupada sin protección proporcionan una idea acerca de la mano de obra que no está en el sector informal pero que a pesar de ello se encuentra en una situación de desprotección laboral. Este indicador hace todavía más visible la condición de precariedad del trabajo juvenil y su deterioro en las situaciones de crisis. Al comparar 2008-2009 la participación en este tipo de ocupaciones se incrementa ligeramente en los varones de 14 a 19 años y en forma más importante, en el grupo entre 20 y 29 años.

Lo anterior indica que la mano de obra juvenil está frente a modalidades de participación en el mercado laboral en las que el trabajo deja de ser una vía de acceso a uno de los derechos laborales esenciales: la protección social. Las cifras de 2009 para todo el país -excluyendo a quienes trabajan en el sector informal ${ }^{21}$ - muestran que el grupo de 14 a 19 años $-44.2 \%$ y $42.2 \%$ respectivamente de los y las jóvenes ocupados- no tienen acceso a instituciones de salud por medio del empleo; en el grupo de 20 a 29 años estas cifras son menores pero todavía superiores al 30\% de ocupados en este grupo de edad (cuadros 1 y 2 ).

\section{El impacto diferencial de la crisis económica según el grado de asalarización de los mercados de trabajo.}

Ahora bien, constatamos -una vez más- la mayor precariedad laboral de la mano de obra muy joven frente a los grupos de mayor edad y observamos el mayor deterioro laboral experimentado por la fuerza de trabajo joven en el contexto de la primera crisis del siglo XXI.

Está pendiente analizar el efecto de la crisis sobre la mano de obra juvenil según el tipo de mercado de trabajo en el que participa. Para ello clasificamos los mercados laborales estatales según el grado de asalarización blación ocupada". Nosotros hablamos de ocupaciones sin protección laboral eliminando de la definición anterior a la población ocupada que labora en el sector informal; este grupo se analiza de forma separada.

${ }^{18}$ Nos referimos a los cambios entre el II trimestre del 2008 y el II trimestre del 2009.

${ }^{19}$ Para análisis del desempleo en México y América Latina, véanse Mesa Romero (2006), Ros (2005).

${ }^{20}$ Tasa de trabajo asalariado se refiere al "porcentaje de la población ocupada que percibe de la unidad económica para la que trabaja un sueldo, salario o jornal, por las actividades realizadas "(INEGI).

${ }^{21} \mathrm{Si}$ se incluye este grupo el porcentaje de las y los trabajadores ocupados de 14 a 19 sin seguro social asciende a un $82 \%$. 
${ }^{22}$ Puesto que nos referimos a una mezcla de actividades urbanas y rurales organizadas en torno a lógicas de reproducción del hogar, debe entenderse esta noción en términos de actividades de subsistencia. El uso de las comillas tiene como propósito enfatizar este rasgo. que presentan, lo cual permite observar, en alguna medida, la heterogeneidad estructural que caracteriza los mercados de trabajo en el país.

En consecuencia se agruparon las 32 entidades federativas en cinco contextos laborales diferenciados de acuerdo con el grado de asalarización de sus mercados de trabajo mediante la aplicación de un análisis de conglomerados. Como se puede observar en el cuadro 3 el grado de asalarización de la población ocupada guarda una estrecha relación con algunos indicadores de desarrollo socioeconómico de las entidades, tales como: el índice de rezago social, la proporción de población rural, los niveles salariales de la población ocupada y el índice de intensidad migratoria hacia los Estados Unidos.

Se espera que las manifestaciones de la crisis económica en la población muy joven varíen de acuerdo con la naturaleza más o menos asalariada de los mercados de trabajo y en relación con el sexo de la mano de obra joven. Por lo general, los niveles de desempleo suelen ser más elevados en los mercados de trabajo donde predomina el empleo asalariado, es decir, allí donde el desarrollo del capitalismo ha implicado una mayor expropiación de medios de producción al factor trabajo. En cambio, en las entidades con menor grado de asalarización, los efectos de la crisis económica deberían propiciar la expansión del empleo en el sector informal22. A su vez, las ocupaciones sin protección social, debido a la gran heterogeneidad de situaciones que abarca (véase nota 14) podrían incrementarse en mercados de trabajo con grados de asalarización diferenciados.

A continuación se compara el deterioro de la situación laboral de la mano de obra más joven en las entidades federativas clasificadas de acuerdo con su mayor o menor grado de asalarización. Se analizan - como un indicador del impacto de la crisis económica- las variaciones porcentuales que se han dado principalmente en tres indicadores: niveles de desempleo, participación en el sector informal y ocupaciones sin protección laboral. Se busca encontrar los contextos labores donde la mano de obra joven ha sido más afectada. También interesa destacar las entidades federativas -al interior de cada contexto- donde el impacto de la crisis en la población joven ha sido más severo. En estos casos, hacemos referencia a lo ocurrido con la población total ocupada en las diferentes entidades para sopesar el 
mayor o menor deterioro en la situación laboral de la mano de obra joven en relación con la PEA total.

\section{Mercados de trabajo con un muy alto grado de asalarización}

Nuevo León, Aguascalientes, Coahuila, Baja California Sur y Baja California, son las entidades, que en orden decreciente, presentan un mayor predominio de trabajo asalariado en el país ${ }^{23}$. Se trata de estados con un alto grado de desarrollo económico y social. Cuentan con bajos niveles de rezago social y de población rural ${ }^{24}$ y con mejores condiciones de vida ${ }^{25}$. Con la excepción de Aguascalientes, todas estas entidades están en regiones del norte del país y se distinguen por un grado medio o bajo de intensidad migratoria hacia Estados Unidos (cuadro 3). La participación económica juvenil en estos estados no es muy elevada, en 2008 solamente en Nuevo León, la población joven presentaba tasas de participación económica por arriba del promedio nacional (cuadro 4).

La crisis económica global acarreó serios problemas laborales para la mano de obra juvenil en los contextos con muy alto grado de asalarización y desarrollo industrial en la región norte del país. Los y las jóvenes de 14 a 19 años enfrentaron elevados incrementos del desempleo y de las ocupaciones sin protección laboral ${ }^{26}$ (cuadro 5 y 6). En 2009 en todas las entidades en este grupo, el desempleo de la mano de obra más joven fue superior al promedio nacional correspondiente y en casi todas, los niveles de desempleo en 2009 están entre los diez más elevados del país ${ }^{27}$. Nótese, además, que en lo relativo a la desprotección laboral de los varones, a pesar del incremento acaecido, en ningún caso los niveles se ubican entre los diez más elevados (cuadro 7).

Para valorar la mayor o menor intensidad de las repercusiones de la coyuntura económica analizada en la situación laboral de la mano de obra juvenil, destacamos las situaciones que nos parecen más críticas sea por afectar tanto a hombres como a mujeres jóvenes, tener consecuencias en los tres indicadores considerados o por sobresalir en algunos de ellos. Nuevo León y Baja California presentan los más elevados incrementos del
${ }^{23}$ Con variaciones entre 74 y $70 \%$ de la PEA total de la entidad federativa en actividades asalariadas (cuadro 3).

${ }^{24}$ En localidades con menos de 5 mil habitantes.

${ }^{25}$ Menor proporción de población ocupada que percibe salarios/ingresos hasta 2 salarios mínimos.

${ }^{26}$ Consideramos como un incremento elevado cuando el indicador analizado se ubica en los primeros diez lugares en el rango del conjunto de las treinta y dos entidades del país.

${ }^{27}$ Las dos excepciones son Baja California Sur para los hombres y Aguascalientes para las mujeres. 
${ }^{28} \mathrm{El} 35.3 \%$ y $24.4 \%$ respectivamente del PIB de Coahuila y Nuevo León se generan en las actividades manufactureras (cifras para 2008 a precios de 2003, INEGI) (cuadro 10).

${ }^{29}$ Datos de la ENOE no presentados en los cuadro desempleo en los hombres y las mujeres en esta franja de edad. Coahuila, a su vez, se distingue por el aumento del desempleo masculino juvenil y sobre todo por una expansión muy acentuada de las ocupaciones sin protección laboral en los hombres y las mujeres jóvenes (cuadro 5 y 6 ).

En Nuevo León y Coahuila el desempleo masculino y femenino en la población joven ya era muy elevado antes de la crisis económica, aspecto que denota la intensificación de una situación ya crítica anteriormente. En 2009, en estas dos entidades este indicador alcanza en los hombres cifras superiores a $19 \%$, entre las mujeres sube a $25 \%$ en Nuevo León y a $17.4 \%$ en Coahuila (cuadros 7 y 8). La expansión del desempleo en estas entidades no es privativa de las personas jóvenes, ha afectado también de modo marcado a la PEA total (cuadro 9). En Nueva León, el impacto de la crisis en la mano de obra juvenil femenina fue muy intenso, además del muy alto nivel de desempleo alcanzado, las mujeres redujeron su tasa de actividad económica; y seguramente se han "refugiado"en la realización de los quehaceres domésticos como suele ocurrir debido a la división sexual del trabajo prevaleciente en nuestra sociedad (cuadro 4).

Estos dos estados castigados por el desempleo se caracterizan por un alto grado de industrialización ${ }^{28}$ donde destaca la producción de maquinarias y equipo y las diferentes ramas del terciario tienen una menor aportación al PIB estatal, sobre todo en Coahuila (cuadros 10 y 11). En uno y en otro caso, la industria manufacturera utiliza en forma importante la mano de obra juvenil. En 2008, 28.5\% de los hombres en Nuevo León y 20.3\% en Coahuila participaba en estas actividades; las cifras correspondientes para las mujeres fueron de $23 \%$ y $16.2 \%$ (cuadros 12 y 13 ).

Llama la atención el aumento del desempleo femenino en Baja California Sur ascendió de 3.6\% a 14.9\% (cuadro 8), despuntando como el incremento más elevado en el país entre 2008 y 2009. En esta entidad la actividad económica se concentra principalmente en el comercio y los servicios de alojamiento temporal y preparación de alimentos y bebidas (cuadros 10 y 11). En consecuencia, en 2008 las mujeres de 14 a 19 años que trabajaban en Baja California Sur lo hacían en $\mathbf{4 7 . 8 \%}$ de los casos en las actividades de servicios (cuadro 13) y gran parte en los servicios de alojamiento y preparación de alimentos vinculados a la actividad turística ${ }^{29}$. Los hombres, a diferencia de 
las mujeres, enfrentaron además una reducción en la tasa de participación económica, esto es, un incremento de la inactividad (cuadro 4).

En los contextos con muy alta asalarización, entre 2008 y 2009, los niveles de informalidad de los y las jóvenes no se incrementaron en forma importante e incluso se redujeron en algunos estados -con excepción de Baja California- (cuadro 5 y 6). En el último año, en casi todos los casos, la participación juvenil en el sector informal se encontraba por debajo del promedio nacional correspondiente (cuadros 7 y 8 ).

Lo acaecido en Baja California merece una mención especial. Estamos frente a un contexto altamente urbanizado, con un peso importante de las industrias manufactureras, en especial de maquinaria y equipo, así como con niveles salariales de la población ocupada superiores al resto de las entendidas federativas (cuadro 3 y 10). Sin embargo, a pesar del alto nivel de desarrollo económico y social del estado -en el contexto nacional-, la situación laboral juvenil se deterioró en forma considerable en los años de la crisis. Además de la fuerte expansión del desempleo, las y los jóvenes ampliaron en forma importante su participación en las ocupaciones sin protección social y los varones incrementaron su presencia en el "sector informal" (cuadros 5 y 6)..$^{30}$ En 2008, en esta entidad, alrededor de 16\% de la mano de obra masculina y femenina más joven participaba en las actividades industriales; la presencia de los hombres en la agricultura ascendía a $18.7 \%$ y más de $50 \%$ de las mujeres estaba en actividades de servicio (cuadros 12 y 13).

Cifras estimadas muestran el fuerte impacto de la crisis en esta entidad, la cual experimentó una reducción del PIB del -10.5\% en 2009. La caída del empleo formal y de los salarios en 2008 y 2009 fue superior al promedio nacional. La recepción de remesas de familiares que se encuentran en el extranjero alcanzó el nivel más bajo en el cuarto semestre de 2008 (Banamex, 2010). En suma, varios factores se conjugan y permiten explicar, en parte, el deterioro de la situación laboral en esta entidad.
${ }^{30}$ En Baja California el aumento del desempleo, informalidad y desprotección laboral no ocurrió solamente en la población joven, se hicieron presentes también en el total de la fuerza laboral de la entidad (cuadro 9). 
${ }^{31}$ Este grupo se caracteriza por contar con proporciones de trabajo asalariado que representan entre 67 y $65 \%$ de la PEA total de la entidad federativa (cuadro 3).

${ }^{32} \mathrm{Al}$ hablar de porcentajes reducidos o elevados nos estamos refiriendo a la comparación con el promedio nacional del indicador o grupo en cuestión.

${ }^{33}$ Dato de la ENOE, no presentado en el cuadro.

\section{Mercados de trabajo con alto grado de asalarización}

Este grupo incluye a nueve entidades ${ }^{31}$ que se caracterizan por una mayor heterogeneidad regional, económica y social. Cuatro se encuentran en las regiones del norte: Tamaulipas, Chihuahua, Sonora y Durango. Con la excepción de este último estado, los tres restantes presentan mejores condiciones económicas y sociales que el promedio nacional. Del centro del país están en este grupo el Distrito Federal, Querétaro y Estado de México; y en el sur: Quintana Roo y Tabasco. Con la excepción de Durango, Querétaro y Tabasco, en todos los demás estados la proporción de población rural es reducida ${ }^{32}$. Asimismo, solamente Durango cuenta con condiciones de vida inferiores al promedio nacional y un muy alto grado de intensidad migratoria a Estados Unidos (cuadro 3). La participación económica juvenil en estos estados tampoco es muy elevada, solamente Tamaulipas y Quintana Roo se distinguen por presentar niveles por arriba del promedio nacional (cuadro 4).

En este contexto laboral, la recesión económica también conllevó incrementos importantes en el desempleo de la mano de obra juvenil. Tamaulipas y Sonora se distinguen por ocupar los primeros lugares en el país en la ampliación del desempleo masculino y femenino juvenil. Quintana Roo, a su vez, sobresale por el aumento del desempleo de los hombres jóvenes y Querétaro por el de las mujeres jóvenes (cuadros 5 y 6 ). En esta última entidad, en $2008,29.2 \%$ de la mano de obra juvenil femenina trabajaba en actividades industriales (cuadro 13), sobre todo en la industria textil y del vestido ${ }^{33}$.

Resalta la situación de Sonora, donde a los elevados incrementos del desempleo se agrega la acentuada expansión de la informalidad juvenil en hombres y mujeres. Es preciso tener presente que en esta entidad federativa la informalidad también se incrementó en forma importante en la población ocupada total (cuadro 9). Para completar el escenario de deterioro laboral en Sonora, los varones redujeron -en forma importante- su participación económica, expandiéndose así la inactividad juvenil masculina. Esta situación contrasta con lo ocurrido en Tamaulipas, Quintana Roo y Querétaro donde aumentó la inactividad femenina (cuadro 4).

Sonora, al igual que Baja California, Coahuila y Nuevo León, cuenta con una importante producción manufacturera de maquinaria y equipos (cuadro 
10). Presenta muy bajo nivel de regazo social y una baja intensidad migratoria a Estados Unidos (cuadro 3). Estas entidades enfrentaron un desplome de su PIB en 2009; aspecto que se asocia a la pérdida de dinamismo de la actividad industrial orientada hacia el mercado externo, a la contracción del empleo formal, de los salarios y del crédito comercial (Banamex, 2009).

En un contexto de contracción económica tan acentuada, no es sorprendente que, a diferencia de lo que esperábamos -a la par de la expansión del desempleo- la presencia de los jóvenes varones en el sector informal se incrementara en forma importante en siete de los nueve estados con alto grado de asalarización. Tamaulipas y Quintana Roo se distinguen no solamente por los elevados incrementos del desempleo masculino sino también por la expansión de la "informalidad" entre los jóvenes varones.

Es sobresaliente lo acontecido en Quintana Roo, donde la mano de obra juvenil masculina presenta un acentuado deterioro en los tres indicadores considerados -desempleo, informalidad y ocupaciones sin protección laboral- mientras las jóvenes más bien se "refugian" en la inactividad. En esta entidad también acaeció un incremento importante del desempleo y de las ocupaciones sin protección laboral en el conjunto de la fuerza laboral (cuadro 9). El Distrito Federal, a su vez, se destaca por el mayor aumento -en el país- de la informalidad juvenil masculina, en la cual laboraba el $48.3 \%$ de los varones de 14 a 19 años que se identificaron como ocupados en el 2009 (cuadros 5 y 7 ).

Tamaulipas, Quintana Roo y el Distrito Federal comparten una baja proporción de población rural, muy baja intensidad migratoria a Estados Unidos y niveles de bienestar superiores al promedio nacional (cuadro 3). Pero se distinguen por su ubicación regional, el tamaño de sus poblaciones y por su especialización productiva. Quintana Roo alberga a Cancún, uno de los principales centros de atracción del turismo en el país. En 2008, 20.4\% del PIB de esta entidad era generado por los servicios de alojamiento temporal y de preparación de alimentos y bebidas (cuadro 11). En la misma fecha estos servicios absorbían alrededor de $13 \%$ de la mano de obra femenina de 14 a 19 años de edad; en el caso de los varones en el mismo tramo de edad, esta cifra asciende a casi 18\% (cuadros 12 y 13).

En contraste, Tamaulipas, sobresale por una más elevada contribución 
del sector de la construcción y la minería al PIB estatal; alrededor de 10\% de los jóvenes varones se ocupaba en la construcción en 2008. El Distrito Federal, por su parte, como sede del gobierno federal, concentra en mayor medida que las demás entidades del país, los servicios financieros y de seguros -actividades que en el 2008 generaron el 14.4\% del PIB de la entidad-, así como los servicios profesionales, de información masiva y las actividades del gobierno (cuadro 11). En 2008, en la capital del país, un poco más de la mitad de los y las jóvenes de 14 a 19 años trabajaban en los servicios, casi 20\% en los servicios de alojamiento y preparación de alimentos (cuadros12 y 13).

Estos resultados ponen de manifiesto que en Tamaulipas, Quintana Roo y el Distrito Federal las repercusiones de la crisis en la mano de obra juvenil han sido más visibles en los varones y se manifiestan de diversas maneras y en diferentes sectores económicos. Se dejaron sentir en la construcción, en la industria del turismo, en las actividades comerciales y en los servicios, sea en forma de desempleo o informalidad. Dada la importancia del turismo en Quintana Roo, la transmisión de la crisis global a la economía local se expresó en la contracción de -7.1\% del PIB estatal. En Tamaulipas la reducción de este indicador fue aún mayor mientras que en el DF la cifra correspondiente se ubicó en torno al promedio nacional (estimación de Banamex, 2009).

Chihuahua requiere una mirada más atenta. En esta entidad -con una presencia acentuada de las actividades manufactureras, en especial la producción de maquinarias y equipos- las repercusiones de la crisis han sido muy distintas en la mano de obra juvenil masculina y femenina. Mientras los hombres sufrieron un aumento de la informalidad, la desprotección laboral y la inactividad, las mujeres se mostraron más afectadas por el desempleo (cuadros 4, 5 y 6). Este mayor deterioro de la situación laboral de la mano de obra juvenil masculina en comparación con la femenina está asociado en cierta medida a las marcadas diferencias en su inserción laboral. En 2008, el 18.3\% de los hombres jóvenes ocupados en esta entidad estaba en la agricultura, $13.5 \%$ en la construcción y $16.6 \%$ en el sector industrial; situación que contrasta con la de las mujeres jóvenes ocupadas, quienes en cerca del $40 \%$ trabajaban en las actividades manufactureras, en especial en las industrias maquiladoras (cuadros 12 y 13). 
Vale detenernos un poco más en las repercusiones de la crisis económica sobre la situación laboral de las mujeres jóvenes en los contextos con alta asalarización. El desempleo de las jóvenes se incrementó en más de la mitad de los estados con alta asalarización. Sonora, Durango, Chihuahua, Querétaro y Tamaulipas sobresalen con los mayores incrementos (cuadro 6). En este último estado las jóvenes ya enfrentaban un alto desempleo desde antes de la crisis pero, a raíz de ésta, la desocupación abierta se dispara hasta alcanzar a poco más de dos décimas partes de la mano de obra más joven -22.8\% en 2009 (cuadro 8).

Durango y Sonora se destacan, además, por presentar incrementos extremadamente altos en la informalidad femenina juvenil ${ }^{34}$. En 2009 entre las jóvenes ocupadas, 50.5\% en Durango y 36.7\% en Sonora estaban en el sector informal (cuadro 8). Apréciese que mientras los hombres expandieron en forma marcada su presencia en actividades informales en siete de los nueve estados considerados, las mujeres lo hicieron solamente en estas dos entidades (cuadros 5 y 6). En Durango, en contraste con Sonora, el deterioro de la situación laboral de las jóvenes transcurre en un escenario caracterizado por una intensa migración hacia Estados Unidos, elevadas proporciones de población rural y con condiciones de vida mucho más precarias (cuadro 3). En esta entidad, la actividad manufacturera también es importante -21.2\% del PIB estatal en 2008- pero está orientada a la producción de bienes de consumo final, sobre todo a la producción de alimentos, bebidas y tabaco; además, las actividades del sector primario representaban en 2008 el 12.3\% del PIB estatal (cuadro 10). En la misma fecha, cerca de $18 \%$ de la mano de obra juvenil femenina trabajaba en las actividades manufactureras, particularmente en la industria textil y del vestido, 34.6\% en el comercio al por menor y solamente $2.5 \%$ en la agricultura (cuadro 13 y datos de la ENOE no incluido en los cuadros).

Asimismo, la mano de obra juvenil femenina en los mercados con alta asalarización fue más afectada que la masculina por la desprotección laboral. ${ }^{35}$ En cuatro de los estados en este grupo (Querétaro, Edo. de México, Tabasco y Distrito Federal), la crisis económica acarreó para las jóvenes un incremento en la precarización de la inserción laboral. Querétaro, cuya economía está orientada a las actividades industriales, comercio y-trans-
${ }^{34}$ Durango, al igual que Sonora, sobresale por los más elevados incrementos de la informalidad en la PEA total (cuadro 9).

${ }^{35}$ Para una discusión sobre feminización de los mercados de trabajo y precariedad laboral véanse: Ariza y Oliveira (2003) y Ariza (2006). 
${ }^{36}$ Los niveles de asalarización varían entre 63 y $58 \%$ de la PEA total (cuadro 3). portes (cuadros 10 y 11), despunta con el más elevado incremento de la desprotección laboral de las jóvenes en el contexto nacional (cuadro 6). En esta entidad, en 2008, 29.2\% de las mujeres ocupadas de 14 a 19 años trabajaban en el sector industrial (cuadro 13), principalmente en la rama textil y del vestido (18.9\%).

En suma varias son las entidades federativas con muy alto o alto grado de asalarización, ubicadas en la región norte del país, donde los o las jóvenes -o ambos- enfrentaron un fuerte deterioro de su situación laboral mediante el incremento del desempleo, de la desprotección laboral o de la informalidad. Las dificultades que han enfrentado la población joven en el mercado de trabajo en el norte del país también se han extendido en gran parte de los casos hacia la PEA total de la entidad. Baja California, Coahuila, Chihuahua, Durango, Nuevo León, Sonora y Tamaulipas son ejemplos de ello. De acuerdo con estimaciones de Banamex, durante el año 2009 el PIB de estas entidades presentó una caída abrupta muy por arriba del promedio nacional de $-6.5 \%$. Coahuila sobresale con una reducción estimada del PIB de -14.4\% y las demás entidades presentan decrementos que van del $-13.6 \%$ en Chihuahua a $-7.8 \%$ en Tamaulipas. Las repercusiones tan intensas de la crisis global en estas entidades se debe, en gran medida, a la importancia que asume la industria manufacturera de exportación en su economía (Banamex, 2009). Se trata no sólo de economías más asalariadas sino también con mayor grado de dependencia del desempeño del mercado estadounidense.

\section{Mercados de trabajo con un grado moderado de asalarización. ${ }^{36}$}

Este contexto laboral es aún más heterogéneo en términos de los niveles de desarrollo, urbanización, condiciones de vida e intensidad migratoria que el anterior (cuadro 3). Aquí se encuentran estados de diferentes regiones del país con muy bajos y altos grados de rezago social, urbanización y emigración: Sinaloa en el noroeste, Jalisco y Colima en el occidente, Tlaxcala, Morelos y Guanajuato en el centro/centro norte y Campeche, Yucatán y Veracruz en el sureste.

A medida que el grado de asalarización de los mercados de trabajo disminuye, el panorama en cuanto a la participación económica juvenil y las consecuencias de la crisis económica en la fuerza de trabajo joven empie- 
zan a cambiar. En este contexto de moderada asalarización, la participación económica de los y las jóvenes es elevada; en más de la mitad de los casos se encontraba, en 2008, por arriba del promedio nacional (cuadro 4). Asimismo, en la mayoría de las entidades de este grupo, los niveles de desempleo juvenil son más reducidos y experimentaron menores incrementos que en los contextos laborales más asalariados. El deterioro de la situación laboral también ha sido diferencial para hombres y mujeres jóvenes.

En el caso de los varones, las manifestaciones de la crisis no son tan claras como en los contextos más asalariados. No se reportan incrementos elevados en el desempleo (10 primeros rangos) entre 2008-2009 e incluso en algunas situaciones este indicador ha disminuido su intensidad. La informalidad de la mano de obra masculina, a su vez, se ha expandido en forma importante solamente en Sinaloa y Guanajuato mientras que las ocupaciones sin protección social en Colima, Tlaxcala y Morelos (cuadro 5).

En 2009, resalta el incremento de la informalidad masculina en Guanajuato. En esta entidad federativa, la industria manufacturera aporta casi $28 \%$ del PIB estatal pero presenta una composición más polarizada que en los estados del norte del país. En este caso, las industrias de capital coexisten con las orientadas al consumo final (cuadro 10) y seguramente, las grandes empresas con las de menor tamaño. Se trata de una entidad con un muy alto grado de intensidad migratoria, un elevado índice de regazo social, mayor peso de población rural y peores niveles de vida que el promedio nacional (cuadro 3). En 2008, 20\% de los varones de 14- 19 años trabajaban en la agricultura y alrededor de $23 \%$ en actividades industriales, principalmente en la industria del cuero (cuadro 12 y ENOE, 2008).

En este contexto de asalarización moderada, en más de la mitad de los casos, la mano de obra femenina juvenil incrementó en forma marcada su participación en el sector informal (cuadro 6). Despuntan Sinaloa y Campeche con incrementos superiores a 15 puntos porcentuales (cuadro 8). Estas dos entidades comparten grados moderados de asalarización, pero presentan notables diferencias en cuanto a su estructura productiva, los grados de rezago social, intensidad migratoria, el peso de la población rural y los niveles salariales de la población. La economía sinaloense gira en torno a las actividades agrícolas y comerciales mientras en Campeche se 
${ }^{37}$ En Campeche, a diferencia de Sinaloa, el incremento de la informalidad fue de igual modo elevado en la PEA total (cuadro 9).

${ }^{38}$ Las cifras exactas van de $53 \mathrm{y}$ $56 \%$ de la PEA de las entidades federativas correspondientes (cuadro3). da un predominio notable de la minería. Además, Sinaloa se distingue por un bajo índice de regazo social y un alto grado de intensidad migratoria; mientras Campeche presenta un alto grado de rezago social, muy baja intensidad migratoria hacia Estados Unidos y un mayor peso de la población rural y con escasos recursos (cuadro 3, 10 y 11). ${ }^{37}$ Todo indica que, a diferencia de lo ocurrido en los contextos más asalariados, el impacto de la crisis en estas entidades con asalarización moderada fue menos severo. Con la excepción de Campeche y Tlaxcala, en todas las demás entidades la reducción estimada del PIB estuvo alrededor o por abajo del promedio nacional (Banamex, 2009).

\section{Los mercados de trabajo con bajo grado de asalarización}

Estos mercados están presentes también en diferentes regiones del país. San Luis Potosí y Zacatecas en el centro norte, Michoacán y Nayarit en el occidente e Hidalgo y Puebla en la región centro. Estos estados, que cuentan con alrededor de la mitad ${ }^{38}$ de la PEA total en actividades asalariadas, presentan porcentajes elevados de población rural y con bajos ingresos. Se distinguen, asimismo, por grados de intensidad migratoria que van de medio a muy alto y con excepción de Nayarit todos exhiben niveles de rezago social que varían de igual modo de medio a muy alto (cuadro 3).

Es un contexto de elevada utilización de mano de obra juvenil, en todos los casos la participación económica de los y las jóvenes se ubica por arriba del promedio nacional (cuadro 4). Casi todas estas entidades presentaron en 2009, una reducción estimada del PIB inferior al promedio nacional, las únicas excepciones fueron Puebla y Nayarit que tuvieron cifras de contracción de la economía de -9.4\% y -7.7\% respectivamente (Banamex, 2009).

En este contexto con bajos grados de asalarización, el deterioro de la situación laboral juvenil fue más acentuado en las mujeres que en los hombres. Estos últimos no experimentaron incrementos elevados en ninguno de los tres indicadores considerados -desempleo, informalidad y desprotección laboral- (cuadro 5). Solamente en Michoacán, el desempleo de los hombres jóvenes se incrementó en 7.1 puntos porcentuales entre 2008 y 
2009 -el cuarto aumento más elevado del conjunto de las entidades federativas-, afectando en 2009 al 10.5\% de la fuerza de trabajo correspondiente (cuadro 7). En 2008, 10.6\% del PIB del estado provenía de la agricultura y $18.4 \%$ del comercio (cuadros 10 y 11). En la misma fecha, estas dos ramas de actividad absorbían, respectivamente 37\% y 15.1\% de la mano de obra juvenil masculina (cuadro 12). Nótese también que en Michoacán la inactividad masculina se incrementó en forma importante, al bajar su tasa de participación económica (cuadro 4).

En el caso de la mano de obra femenina juvenil, la crisis estuvo asociada -al igual que en el contexto de asalarización moderada- a una expansión importante de los niveles de informalidad en San Luis Potosí, Hidalgo y Michoacán (cuadro 6). En 2009, 31.9\%, 34.0\% y 40.8\% de las jóvenes de 14 a 19 años en cada una de estas entidades respectivamente se ocupaban en el sector informal (cuadro 8). Estos tres estados, a pesar de las diferencias en su estructura económica -Michoacán más orientado a actividades agrícolas o comerciales, y los otros dos a actividades industriales de consumo final- comparten un alto grado de rezago social, así como proporciones de población rural y de bajos ingresos superiores al promedio nacional. Michoacán e Hidalgo también presentan un muy alto o alto grado de intensidad migratoria (cuadro 3, 10 y 11). ${ }^{39}$ Este último estado se distingue, además, por la mayor reducción de la actividad económica de la mano de obra juvenil femenina, esto es, por el mayor incremento de la inactividad de las jóvenes (cuadro 4).

Mercados de trabajo con un muy bajo grado de asalarización. En Chiapas, Guerrero y Oaxaca, estados de la región sur-pacífico, la proporción de trabajo asalariado es aún más reducida -entre 41 a $43 \%$ de la PEA total- y los índices de rezago social son los más elevados del país. Asimismo, se encuentran entre los estados con mayores proporciones de población rural y de más bajos ingresos (cuadro 3). La participación económica de los hombres jóvenes es elevada, en los tres casos se encuentra entre los diez mayores niveles del país, mientras que con las mujeres jóvenes esto ocurre solamente en Oaxaca (cuadro 4).

En estos contextos de alta privación relativa, la mano de obra muy joven muestra niveles e incrementos de desempleo muy por debajo del prome-
${ }^{39} \mathrm{~A}$ diferencia de San Luis Potosí y Michoacán, en Hidalgo el incremento del desempleo fue elevado más bien en la PEA total (cuadro 9). 
${ }^{40}$ En la PEA total de desprotección laboral también aumentó en forma importante en este estado (cuadro 9). dio nacional (cuadros 7 y 8 ). Oaxaca y Guerrero, entidades orientadas a la agricultura, comercio y servicios, se caracterizan, además, por grados medios y altos de intensidad migratoria. Ya desde antes de la crisis económica, la mano de obra masculina y femenina muy joven se distinguía por una alta participación en el sector informal y en ocupaciones sin protección social. En 2009, en Oaxaca, 49.5\% de las mujeres y 35.3\% de los hombres jóvenes estaban en el sector informal y las ocupaciones sin protección laboral ascendían a 32.0 y $34.9 \%$ en las y los jóvenes respectivamente (cuadros 7 y 8). En Guerrero, este último tipo de ocupaciones se expandió aún más en los varones jóvenes alcanzando a 67.2\% en 2009 (cuadro 7) ${ }^{40}$. En el mismo año, esta entidad enfrentó una reducción estimada de -4.9\% del PIB, a diferencia de Chiapas y Oaxaca, que por su baja integración a la economía internacional, fueron menos afectadas por la crisis global, presentando incluso un ligero crecimiento estimado del PIB en 2009 (Banamex, 2009).

\section{Conclusiones}

La precariedad laboral de la mano de obra juvenil ha persistido en el tiempo, haciendo evidente la capacidad diferencial de integración que exhiben los mercados laborales. La mano de obra más joven -14 a 19 años- se encontraba en una situación de precariedad laboral extrema, con porcentajes importantes en actividades informales y sin protección social desde antes de la actual crisis económica. Este patrón de participación laboral precaria se acentúa en el contexto de la crisis de la economía globalizada, a causa del aumento del desempleo.

En el ámbito nacional, las consecuencias laborales de la crisis se manifiestan en forma más intensa en la población masculina y femenina más joven, observando que la reducción de las tasas de participación y el aumento del desempleo son más acentuados entre ellos. En cambio el subempleo ha afectado con mayor severidad a la población adulta, en particular al grupo que se encuentra entre 50 y 59 años.

En lo relativo a los indicadores de precariedad, las mujeres de 14 a 19 años presentan un mayor incremento en el sector informal; mientras en los 
hombres el grupo más afectado por la desprotección laboral es el de 20 a 29 años. La marcada precariedad del trabajo juvenil -14 a 19 años- acontece en un contexto de elevado déficit general en materia de calidad del empleo, mismo que afecta a amplios contingentes de la fuerza de trabajo. Esto permite fundamentar empíricamente la afirmación de que la tensión entre la dinámica de inclusión y exclusión laboral ha tendido a resolverse, en el modelo de desarrollo globalizador, en favor de esta última. No causa sorpresa que, en este contexto, la fuerza de trabajo juvenil constituya el grupo más vulnerable, debido a su menor experiencia laboral, capital humano y poder de negociación laboral. ${ }^{41}$

Ahora bien, los promedios nacionales ocultan la diversidad de situaciones que acontecen en el interior de México. Mediante un análisis meticuloso de varios contextos laborales mostramos que el impacto de la crisis económica sobre la mano de obra juvenil se ha expresado en forma diferenciada a lo largo del país. Variaciones que dependen de la naturaleza más o menos asalariada de los mercados de trabajo, de las características de la estructura productiva de las entidades federativas así como del mayor o menor grado de integración a la economía mundial. Además, constatamos que la repercusión de la crisis económica es diferencial según la condición de hombre o mujer de la fuerza de trabajo juvenil.

En términos generales, la expansión del desempleo se ha concentrado en los contextos laborales más asalariados, urbanizados e industrializados. Estos contextos fueron los más afectados por la crisis global a raíz de su mayor integración e dependencia de los Estados Unidos. En los contextos con muy alto grado de asalarización, la expansión del desempleo juvenil, al igual que el del conjunto de la fuerza de trabajo, fue generalizada afectando la mayoría de los estados del norte del país. La participación en ocupaciones no protegidas se concentró, de igual modo, en estos contextos laborales. En Baja California, a diferencia de las demás entidades, la situación de los jóvenes varones se tornó aún más crítica; ellos presentaron un deterioro en los tres indicadores considerados (desempleo, informalidad y desprotección).

En contraste, en los contextos con alta asalarización, el impacto de la crisis -al contrario de lo que esperábamos- además de traer la expansión
${ }^{41}$ Para estudios sobre la degradación de la condición laboral, la precariedad del trabajo y los procesos de exclusión social véanse Castel, Robert (1999), Weller (2001), Pérez Sáinz (2003), Pérez Sáinz y Mora Salas (2004 y 2007), Rojas García y Salas Páez. 2007. 
del desempleo juvenil, se ha hecho visible también por el incremento de la informalidad masculina, que alcanzó su mayor expansión en el Distrito Federal. Sonora, a su vez, se destaca por un elevado incremento tanto del desempleo como de la informalidad de la mano de obra juvenil -masculina y femenina-. Tamaulipas y Quintana Roo sobresalen por los aumentos elevados del desempleo y de la informalidad en los varones. Nótese que en Quintana Roo, al igual de Baja California, la mano de obra juvenil masculina estuvo expuesta a incrementos considerables tanto del desempleo y de la informalidad como de la desprotección laboral; aspecto que denota la vulnerabilidad de las economías industriales de exportación y de las actividades vinculadas al turismo frente a la crisis económica global.

La mano de obra joven en los contextos con un grado moderado y bajo de asalariazación fue castigada más bien por el incremento de la informalidad femenina en la mitad o más de la entidades consideradas. Despuntan Sinaloa y Campeche con la mayor expansión en el contexto nacional.

En los contextos con muy bajo grado de asalariazación, las consecuencias de la crisis fueron menos severas -tanto en términos de reducción del PIB como de deterioro de la situación laboral de la mano de obra joven de acuerdo con los indicadores utilizados. En las entidades que conforman este grupo los niveles de desempleo son muy reducidos. En un escenario caracterizado por niveles de desarrollo económico y social extremadamente bajos, con más de la mitad de la población en áreas rurales y con cifras iguales o superiores a $65 \%$ de la población ocupada con ingresos hasta dos salarios mínimos, la mano de obra juvenil migra a otras entidades o a Estados Unidos y los que se quedan no tienen más alternativa que incorporase en el sector informal o en ocupaciones precarias sin protección social. La economía de la subsistencia se impone como modelo de vida, con su consabida capacidad de adaptación a coyunturas recesivas.

Estos resultados nos llevan a reflexionar acerca de las posibilidades ocupacionales futuras de los y las jóvenes mexicanos. En la coyuntura actual caracterizada por la caída del crecimiento económico, aumento de los niveles de pobreza y escalada de la violencia vinculada al crimen organizado, la situación laboral de la población joven, que ya era preocupante desde antes, requiere de atención inmediata. Los contextos labores con más alto 
grado de asalarización han perdido su potencial de integración de la mano de obra joven en empleos de mejor calidad. A su vez, en los contextos con muy bajo grado asalarización, los y las jóvenes no buscan trabajo debido a que ni siquiera las oportunidades de instalarse en la informalidad se han expandido. La inactividad forzada parece ser una condición que podría estar adquiriendo mayor importancia en estos casos.

Los y las jóvenes que a temprana edades trabajan se exponen a situaciones de desempleo, informalidad, precariedad o inactividad forzada y tienen por adelante un futuro incierto y carente de protección social. Ellos se encuentran fuera del sistema escolar, excluidos de los sectores más dinámicos de la economía y sin condiciones de establecerse por su cuenta en forma adecuada. La falta de oportunidades y la incertidumbre laboral constituye un rasgo característico de su cotidianidad. Las posibles repercusiones de situaciones de exclusión laboral en la trayectoria futura de la población joven pueden llegar a ser muy elevadas. Cuando la escuela y el trabajo pierden su potencial de fungir como ámbitos de integración social de la población joven, es decir, cuando el sistema escolar no tiene capacidad de absorber y retener a las y los jóvenes, y los mercados de trabajo no les ofrecen empleos suficientes, ni mucho menos empleos de calidad, pocas son las vías alternas legítimas de que ellos disponen para tener acceso a los derechos ciudadanos. En este contexto, la reproducción intergeneracional de las desigualdades laborales encuentra, tierra fértil para su propagación.

\section{Referencias bibliográficas}

Ariza, Marina (2006), “Mercados de trabajo urbanos y desigualdad de género en México a principios del siglo XXI" en Enrique de la Garza y Carlos Salas (coords.), La situación del trabajo en México, pp. 377-412, UAM-IETCASSI-Plaza y Valdés, México.

Ariza, Marina y Orlandina de Oliveira (2003), "Tendencias y contratendencias de la feminización en México" en Fernando Pozos (coord.), La vulnerabilidad laboral del modelo exportador en México. pp. 143-190, Universidad de Guadalajara, Centro Universitario de Ciencias Sociales y Humanidades, México. 
Banco Nacional de México (BANAMEX, 2009), México, indicadores regionales de actividad económica, División de Estudios Económicos y Sociales, México.

Boltvinik, Julio y Araceli Damián (2003), “Derechos humanos y medición oficial de la pobreza en México", Papeles de Población, enero-marzo, núm. 35, pp. 101-136.

Camarena Córdova, R.M (2005), “Los jóvenes y el trabajo” en E.M. Navarrete (coord.), Los jóvenes ante el siglo XXI, pp. 95-133, El Colegio Mexiquense, México.

Castel, Robert (1999), “Vulnerabilidad social, exclusión: la degradación de la condición salarial” en Jorge Carpio e Irene Novacovsky (comps.), De igual a igual. El desafío del Estado ante los nuevos problemas sociales, Fondo de Cultura Económica, Buenos Aires.

Castillo, Dídimo (2008), Precarización del empleo urbano asalariado privado en Panamá. Determinantes sociodemográficos y socio-laborales, tesis para optar por el grado de Doctor en Ciencias Sociales con especialidad en Estudios de Población, Centro de Estudios Demográficos y Urbanos, El Colegio de México, México.

CEPAL (2009), Crisis en los mercados laborales y respuestas contracíclicas, Boletín CEPAL/OIT, Coyuntura laboral en América Latina y el Caribe.

CEPAL (2009), Panorama social de América Latina, CEPAL, Santiago de Chile.

CONEVAL (2005), Mapas de pobreza por ingresos y rezago social 2005, CONEVAL.

CONAPO (2000), Índice de intensidad migratoria: México-Estados Unidos 2000, CONAPO.

(2006), Índice de marginación social 2005, CONAPO.

Contreras Molotla, Felipe (2003) Vulnerabilidad laboral de los trabajadores 
asalariados en México, 2000, tesis para optar por el grado de Maestro en Población, FLACSO, México.

Cortés, Fernando y Rosa María Rubalcava (1991), Autoexplotación forzada y equidad por empobrecimiento, El Colegio de México, México.

De la Garza, Enrique (1990), “Reconversión industrial y cambios en el patrón de relaciones labores en México" en A. Anguiano (comp.), La modernización de México, pp.315-362, UAM Xochimilco, México.

Encuesta Nacional de Ocupación y Empleo (2008, 2009), INEGI, México. García, Brígida (1999), "Los problemas laborales de México a principios del siglo XXI", Papeles de población, julio-septiembre, núm. 21, pp. 9-19.

García, Brígida y Orlandina de Oliveira (1994), Trabajo femenino y vida familiar en México, El Colegio de México, México.

Hernández Laos, Enrique y Jorge Velázquez (2003), Globalización, desigualdad y pobreza. Lecciones de la experiencia Mexicana, Plaza y Valdez Editores, México.

Hernández Laos, Enrique, Nora Garro e Ignacio Llamas (2000), Productividad y mercado de trabajo en México, Plaza y Valdés editores / UAM-Iztapalapa, México.

Hernández Pérez, Víctor (2007), Panorama del empleo juvenil en México: situación actual y perspectivas, Documento de Trabajo núm. 21, Centro de Estudios Sociales y de Opinión Pública, México.

Meza Romero, Patricia (2006), “El desempleo de los jóvenes en México, 2000-2004", documento inédito, El Colegio de México, México.

Mora Salas, Minor (2009), Ajuste y empleo: tendencias de precarización del trabajo asalariado, El Colegio de México, México. 
Mora Salas, Minor y Orlandina de Oliveira (2009), “El desafío de la inclusión frente a las tendencias de exclusión laboral: El empleo precario en dos países latinoamericanos", Revista Sociología del Trabajo, núm. 66.

Navarrete López, Emma Liliana (2001), Juventud y trabajo: un reto para principios de siglo, El Colegio Mexiquense, México.

Oliveira, Orlandina de (1989), “Empleo femenino en México en tiempos de recesión económica: tendencias recientes" en Jennifer Cooper, Teresita de Barbieri, et al. (comps.), Fuerza de trabajo urbana en México. Características y tendencias, pp. 29-66, UNAM / Miguel Ángel Porrúa, México.

(2006), “Jóvenes y precariedad laboral en México", Papeles de Población, núm. 49 pp. 37-73.

(2009), "El trabajo juvenil en México a principios de siglo xxi”, Renglones, núm. 61 (septiembre 2009-febrero 2010) Disponible en http:// revistarenglones.iteso. $\mathrm{mx} /$ tablacontenidos.php.

Pérez Sáinz, Juan Pablo (2003), “Exclusión laboral en América Latina: viejas y nuevas tendencias", Sociología del trabajo, núm. 47, pp. 107-138.

Pérez Sáinz, Juan Pablo y Minor Mora Salas (2004), "De la oportunidad del empleo formal al riesgo de exclusión laboral. Desigualdades estructurales y dinámicas en los mercados latinoamericanos de trabajo", Alteridades, año 14, núm. 28, julio-diciembre, pp. 37-49.

2007, La persistencia de la miseria en Centroamérica. Una mirada desde la exclusión social, FLACSO, San José.

Quintana, Enrique (1996), “Trabajar sobre los dos México: el empleo juvenil” en Rafael Cordera, José Luis Victoria y Ricardo Becerra (coords.), México joven. Políticas y propuestas para la discusión, pp. 111-116, UNAM, México.

Rendón, Teresa y Carlos Salas (1996), “Empleo juvenil en México. Situación 
actual y tendencias", Jóvenes, año 1, núm. 1, julio-septiembre, pp. 34-45. (2000), "Educación y empleo juvenil” en José Antonio Pérez Islas (coord.), Jóvenes: Una evaluación del conocimiento. La investigación sobre Juventud en México 1986-1999 Tomo I, pp. 13-58, Instituto Mexicano de la Juventud, Colección JóVENES núm. 5, tomo 1, México.

Rojas García, Georgina y Carlos Salas Páez (2007), “La precarización del empleo en México, 1995-2004", Revista Latinoamericana de Estudios del Trabajo, núm. 19, pp 39-78.

Salas, Carlos (2007), “Empleo y trabajo en México, 2001-2006. Un balance inicial", Revista del Trabajo, núm. 3, pp. 137-160.

Weller, Jürgen, (2001), Procesos de exclusión e inclusión laboral: la expansión del empleo en el sector terciario. Serie Macroeconomía del desarrollo, núm. 6, División de desarrollo económico, CEPAL, Santiago de Chile. 
CUADRO 1

TASAS DE DIVERSOS INDICADORES SELECCIONADOS SEGÚN EDAD Y SEXO'1 2008-2009

HOMBRES

\begin{tabular}{|c|c|c|c|c|c|c|c|c|c|c|c|c|}
\hline \multirow[t]{2}{*}{ Grupos de edad } & \multicolumn{2}{|c|}{ Participación } & \multicolumn{2}{|c|}{ Asalarización } & \multicolumn{2}{|c|}{ Desempleo } & \multicolumn{2}{|c|}{ Suboempleo } & \multicolumn{2}{|c|}{ Informalidad } & \multicolumn{2}{|c|}{ Desprotección } \\
\hline & 2008 & 2009 & 2008 & 2009 & 2008 & 2009 & 2008 & 2009 & 2008 & 2009 & 2008 & 2009 \\
\hline 14 a 19 años & 43,0 & 40,4 & 64,0 & 63,0 & 6,3 & 9,7 & 6,2 & 9,8 & 32,4 & 32,5 & 42,9 & 44,2 \\
\hline 20 a 29 años & 86,3 & 85,0 & 75,3 & 74,8 & 5,0 & 7,7 & 7,0 & 11,2 & 24,5 & 24,8 & 28,7 & 31,4 \\
\hline 30 a 39 años & 96,3 & 95,9 & 65,0 & 64,9 & 2,5 & 4,3 & 8,0 & 12,7 & 25,9 & 26,7 & 24,0 & 24,7 \\
\hline 40 a 49 años & 95,5 & 94,9 & 56,2 & 58,1 & 2,1 & 4,1 & 7,9 & 12,4 & 27,5 & 28,1 & 22,2 & 22,7 \\
\hline 50 a 59 años & 89,5 & 88,6 & 49,4 & 50,0 & 2,3 & 3,9 & 7,4 & 12,2 & 26,9 & 28,2 & 27,2 & 26,1 \\
\hline 60 y más & 54,5 & 51,3 & 31,4 & 31,9 & 1,5 & 2,8 & 7,5 & 11,1 & 28,1 & 29,0 & 42,0 & 43,1 \\
\hline Total & 78,3 & 76,8 & 60,4 & 60,7 & 3,3 & 5,4 & 7,4 & 11,8 & 26,9 & 27,5 & 28,6 & 29,4 \\
\hline
\end{tabular}

MUJERES

\begin{tabular}{|c|c|c|c|c|c|c|c|c|c|c|c|c|}
\hline 14 a 19 años & 21,6 & 19,7 & 66,0 & 65,0 & 8,2 & 10,4 & 4,2 & 7,5 & 30,9 & 33,5 & 41,2 & 42,2 \\
\hline 20 a 29 años & 49,0 & 48,0 & 75,6 & 75,9 & 6,8 & 8,3 & 5,2 & 9,4 & 20,2 & 20,1 & 30,7 & 31,4 \\
\hline 30 a 39 años & 53,9 & 53,6 & 64,5 & 65,5 & 3,2 & 4,2 & 6,4 & 10,4 & 26,5 & 27,7 & 24,7 & 24,2 \\
\hline 40 a 49 años & 55,8 & 55,1 & 61,0 & 61,3 & 1,9 & 2,6 & 5,9 & 10,4 & 28,7 & 28,2 & 21,7 & 22,1 \\
\hline 50 a 59 años & 43,3 & 43,7 & 48,3 & 49,0 & 1,4 & 1,6 & 6,3 & 11,6 & 35,4 & 36,7 & 23,3 & 21,8 \\
\hline 60 y más & 19,1 & 18,1 & 25,8 & 29,2 & 0,7 & 1,2 & 8,6 & 9,5 & 53,1 & 53,0 & 23,1 & 23,6 \\
\hline Total & 42,0 & 41,2 & 62,1 & 62,5 & 3,9 & 4,8 & 5,9 & 10,1 & 28,6 & 29,2 & 26,5 & 26,4 \\
\hline
\end{tabular}

${ }^{1}$ La definicion de cada uno de los indicadores se encuentran en el texto.

Fuente: Elaborados con base en datos de la ENOE, segundo semestre 2008 y 2009. 
CUADRO 2

DIFERENCIAS PORCENTUALES EN LOS INDICADORES

SELECCIONADOS POR EDAD Y SEXO 2008-2009'1

HOMBRES

\begin{tabular}{|l|c|c|c|c|c|c|}
\hline Grupos de edad & Participación & Asalarización & Desempleo & Subempleo & Informalidad & Desprotección \\
\hline 14 a 19 años & $-2,7$ & $-1,0$ & 3,4 & 3,7 & 0,1 & 1,4 \\
20 a 29 años & $-1,3$ & $-0,5$ & 2,7 & 4,2 & 0,3 & 2,6 \\
30 a 39 años & $-0,5$ & $-0,1$ & 1,7 & 4,7 & 0,8 & 0,7 \\
40 a 49 años & $-0,6$ & 1,9 & 2,0 & 4,4 & 0,7 & 0,4 \\
50 a 59 años & $-0,9$ & 0,6 & 1,6 & 4,9 & 1,3 & $-1,1$ \\
60 años y más & $-3,3$ & 0,5 & 1,3 & 3,6 & 1,0 & 1,1 \\
Total & $\mathbf{- 1 , 5}$ & $\mathbf{0 , 2}$ & $\mathbf{2 , 1}$ & $\mathbf{4 , 4}$ & $\mathbf{0 , 6}$ & $\mathbf{0 , 8}$ \\
\hline
\end{tabular}

MUJERES

\begin{tabular}{|l|c|c|c|c|c|c|}
\hline Grupos de edad & Participación & Desempleo & Subempleo & Asalarización & Informalidad & Desprotección \\
\hline 14 a 19 años & $-1,9$ & 2,2 & 3,3 & $-1,0$ & 2,6 & 1,0 \\
20 a 29 años & $-1,0$ & 1,5 & 4,2 & 0,3 & $-0,1$ & 0,7 \\
30 a 39 años & $-0,2$ & 0,9 & 4,1 & 1,0 & 1,1 & $-0,5$ \\
40 a 49 años & $-0,6$ & 0,7 & 4,5 & 0,3 & $-0,5$ & 0,4 \\
50 a 59 años & 0,3 & 0,2 & 5,3 & 0,7 & 1,3 & $-1,6$ \\
60 años y más & $-1,0$ & 0,5 & 0,9 & 3,4 & $-0,1$ & 0,5 \\
Total & $\mathbf{- 0 , 8}$ & $\mathbf{0 , 9}$ & $\mathbf{4 , 1}$ & $\mathbf{0 , 4}$ & $\mathbf{0 , 6}$ & $\mathbf{- 0 , 2}$ \\
\hline
\end{tabular}

${ }^{1}$ La definicion de cada uno de los indicadores se encuentran en el texto.

Fuente: Elaborados con base en los datos de la ENOE segundo semestre 2008 y 2009. 
CUADRO 3

CARACTERÍSTICAS DEMOGRÁFICAS Y SOCIOECONÓMICAS DE LAS ENTIDADES FEDERATIVAS

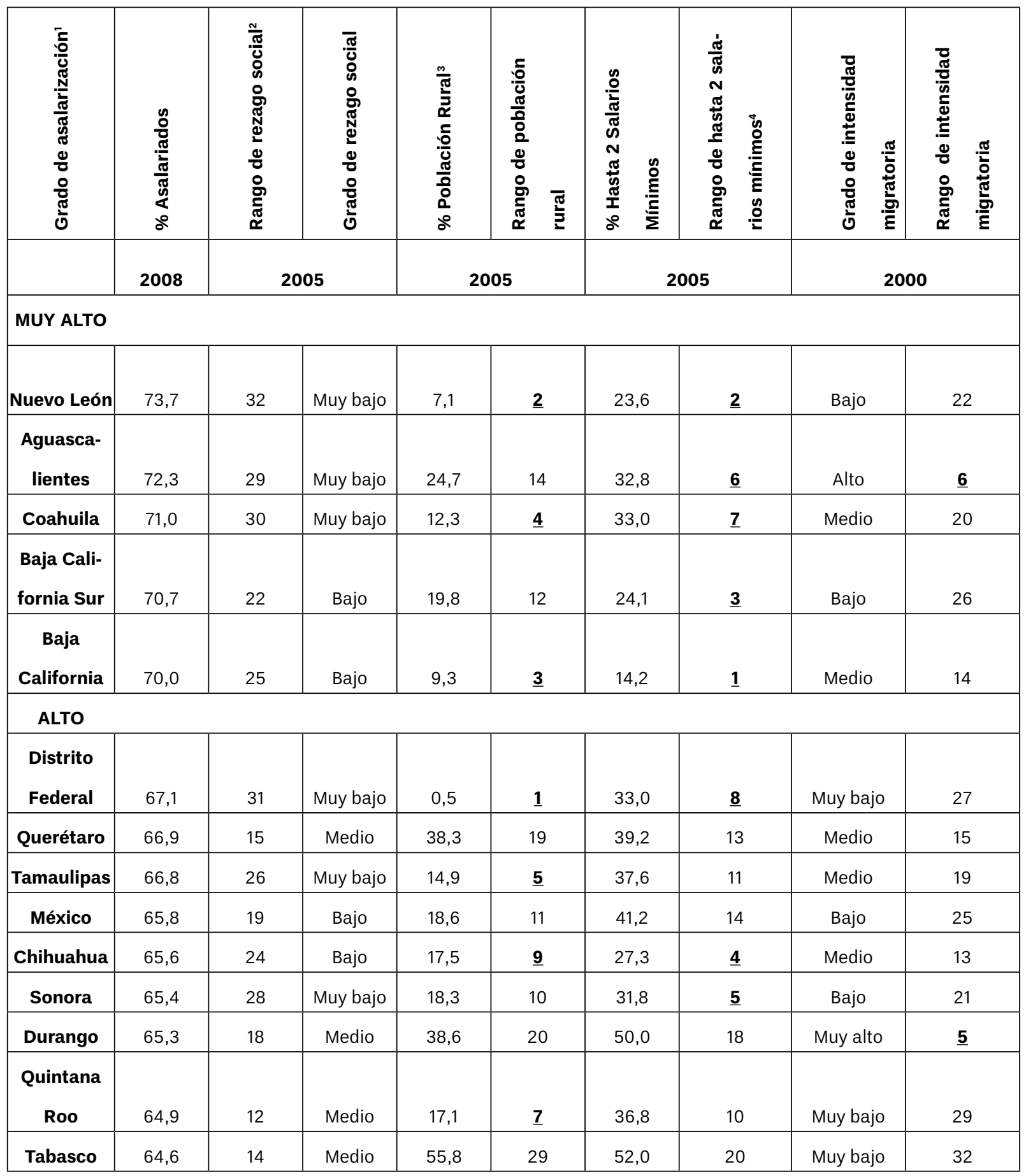




\section{CUADRO 3 (CONTINUACIÓN)}

\section{CARACTERÍSTICAS DEMOGRÁFICAS Y SOCIOECONÓMICAS DE LAS ENTIDADES FEDERATIVAS}

\begin{tabular}{|c|c|c|c|c|c|c|c|c|c|}
\hline 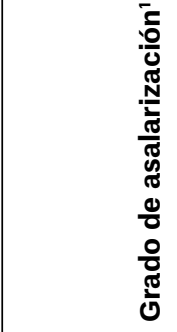 & 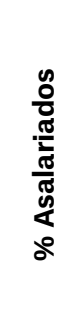 & 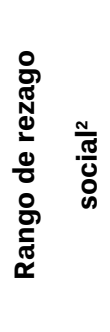 & 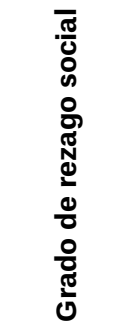 & $\begin{array}{l}\frac{m}{\pi} \\
\frac{m}{7} \\
\frac{0}{\alpha} \\
\frac{0}{0} \\
\frac{0}{0} \\
\frac{\pi}{0} \\
0 \\
0 \\
0\end{array}$ & 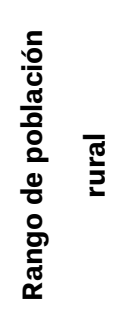 & 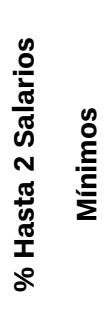 & 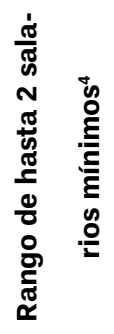 & 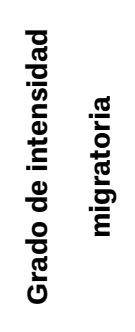 & 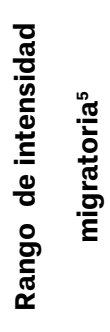 \\
\hline & 2008 & \multicolumn{2}{|c|}{2005} & \multicolumn{2}{|c|}{2005} & \multicolumn{2}{|c|}{2005} & \multicolumn{2}{|c|}{2000} \\
\hline \multicolumn{10}{|l|}{ MODERADO } \\
\hline Jalisco & 62,9 & 23 & Bajo & 17,4 & 8 & 34,7 & 9 & Alto & 7 \\
\hline Colima & 61,7 & 27 & Muy bajo & 15,8 & 6 & 42,7 & 15 & Alto & 8 \\
\hline Sinaloa & 61,5 & 21 & Bajo & 35,3 & 18 & 45,0 & 17 & Alto & 9 \\
\hline Tlaxcala & 60,8 & 13 & Medio & 40,3 & 22 & 62,6 & 28 & Bajo & 24 \\
\hline Morelos & 60,0 & 17 & Medio & 22,9 & 13 & 37,8 & 12 & Alto & 10 \\
\hline Guanajuato & 60,0 & 11 & Alto & 34,5 & 17 & 44,7 & 16 & Muy alto & 3 \\
\hline Campeche & 59,0 & 9 & Alto & 32,0 & 16 & 55,1 & 22 & Muy bajo & 30 \\
\hline Yucatán & 58,4 & 8 & Alto & 27,4 & 15 & 63,0 & 29 & Muy bajo & 28 \\
\hline Veracruz & 58,0 & 5 & Alto & 46,7 & 26 & 58,4 & 25 & Bajo & 23 \\
\hline \multicolumn{10}{|l|}{ BAJO } \\
\hline SLP & 56,1 & 10 & Alto & 41,2 & 24 & 56,1 & 24 & Medio & 17 \\
\hline Hidalgo & 54,4 & 7 & Alto & 57,3 & 30 & 61,6 & 27 & Alto & 12 \\
\hline Nayarit & 53,9 & 20 & Вајо & 41,7 & 25 & 51,7 & 19 & Muy alto & 4 \\
\hline Puebla & 53,5 & 4 & Muy alto & 39,0 & 21 & 61,3 & 26 & Medio & 18 \\
\hline Zacatecas & 53,2 & 16 & Medio & 50,6 & 28 & 54,3 & 21 & Muy alto & 1 \\
\hline Michoacán & 52,9 & 6 & Alto & 40,5 & 23 & 55,8 & 23 & Muy alto & 2 \\
\hline \multicolumn{10}{|l|}{ MUY BAJO } \\
\hline Chiapas & 42,8 & 1 & Muy alto & 58,5 & 31 & 78,1 & 32 & Muy bajo & 31 \\
\hline Guerrero & 42,4 & 2 & Muy alto & 50,5 & 27 & 65,0 & 30 & Alto & 11 \\
\hline Oaxaca & 41,3 & 3 & Muy alto & 61,3 & 32 & 69,7 & 31 & Medio & 16 \\
\hline
\end{tabular}

'El grado de asalarización de la PEA total (INEGI, ENOE, 2008).

${ }^{2}$ Ordenamiento de las 32 entidades federativas según el índice de regazo social. El valor 1 es el de menor regazo social.

${ }^{3}$ Ordenamiento de las 32 entidades federativas según el porcentaje de población en localidades con menos de 5 mil habitantes.

El valor 1 es el de menor proporción de población rural.

${ }^{4}$ Ordenamiento de las 32 entidades federativas según el porcentaje de población que recibe hasta dos salarios mínimos mensuales.

El valor 1 es el de menor proporción de trabajadores con hasta 2 salarios mínimos.

${ }^{5}$ Ordenamiento de las 32 entidades federativas según el índice de intensidad migratria. El valor 1 es el de mayor intensidad migratoria. 
CUADRO 4

TASAS DE PARTICIPACIÓN EN LA ACTIVIDAD ECONOMICA DE LA PEA JOVEN (14-19 AÑOS) Y LAS DIFERENCIAS PORCENTUALES ENTRE DE DICHAS TASAS POR SEXO Y ENTIDAD FEDERATIVA, ENTRE 2008-2009

\begin{tabular}{|c|c|c|c|c|c|c|c|c|}
\hline \multirow{3}{*}{$\begin{array}{l}\text { Grado de } \\
\text { Asalización' }\end{array}$} & \multicolumn{4}{|c|}{ HOMBRES } & \multicolumn{4}{|c|}{ MUJERES } \\
\hline & \multicolumn{2}{|c|}{ Nivel } & \multicolumn{2}{|c|}{ 2008- 2009} & \multicolumn{2}{|c|}{ Nivel } & \multicolumn{2}{|c|}{ 2008- 2009} \\
\hline & 2008 & 2009 & Diferencia & Rango $^{2}$ & 2008 & 2009 & Diferencia & Rango \\
\hline \multicolumn{9}{|l|}{ MUY ALTO } \\
\hline Nuevo León & 43,0 & 41,1 & $-1,9$ & 16 & 28,9 & 25 & $-3,9$ & $\underline{8}$ \\
\hline Aguascalientes & 42,3 & 35,6 & $-6,7$ & $\underline{9}$ & 20,8 & 16,6 & $-4,2$ & $\underline{7}$ \\
\hline Coahuila & 40,8 & 37,8 & $-3,0$ & 15 & 21,1 & 20,6 & $-0,5$ & 21 \\
\hline Baja California Sur & 45,5 & 38,8 & $-6,7$ & $\underline{8}$ & 20,2 & 21,1 & 1,0 & 23 \\
\hline Baja California & 34,0 & 33,2 & $-0,8$ & 20 & 18,3 & 17,8 & $-0,5$ & 20 \\
\hline \multicolumn{9}{|l|}{ ALTO } \\
\hline Distrito Federal & 25,8 & 24,6 & $-1,2$ & 19 & 16,3 & 13,3 & $-3,0$ & 14 \\
\hline Querétaro & 35,4 & 32,2 & $-3,2$ & 14 & 23,1 & 19,8 & $-3,3$ & $\underline{10}$ \\
\hline Tamaulipas & 43,6 & 43,7 & 0,1 & 25 & 22,7 & 19,2 & $-3,5$ & $\underline{9}$ \\
\hline México & 35,0 & 34,4 & $-0,6$ & 22 & 18,4 & 17 & $-1,4$ & 18 \\
\hline Chihuahua & 41,5 & 28,3 & $-13,2$ & $\underline{1}$ & 14,8 & 12,1 & $-2,7$ & 15 \\
\hline Sonora & 36,6 & 29,2 & $-7,4$ & $\underline{4}$ & 18,7 & 19,1 & 19,1 & 32 \\
\hline Durango & 38,5 & 41,0 & 2,5 & 30 & 15,7 & 16,7 & 1,0 & 25 \\
\hline Quintana Roo & 49,1 & 47,3 & $-1,8$ & 17 & 30,5 & 23,6 & $-6,9$ & $\underline{5}$ \\
\hline Tabasco & 41,3 & 40,8 & $-0,5$ & 23 & 14,7 & 19,4 & 4,7 & 31 \\
\hline \multicolumn{9}{|l|}{ MODERADO } \\
\hline Jalisco & 53,3 & 47,0 & $-6,3$ & $\underline{10}$ & 30,4 & 28,9 & $-1,5$ & 17 \\
\hline Colima & 52,5 & 54,3 & 1,8 & 29 & 31,5 & 24,5 & $-7,0$ & $\underline{4}$ \\
\hline Sinaloa & 42,1 & 40,4 & $-1,7$ & 18 & 27,5 & 20,3 & $-7,2$ & $\underline{3}$ \\
\hline Tlaxcala & 42,3 & 46,0 & 3,7 & 31 & 22,8 & 26,1 & 3,3 & 29 \\
\hline Morelos & 40,8 & 42,4 & 1,6 & 28 & 20,4 & 17,3 & $-3,1$ & 13 \\
\hline Guanajuato & 48,1 & 43,8 & $-4,3$ & 13 & 23,4 & 27,1 & 3,7 & 30 \\
\hline Campeche & 45,9 & 47,1 & 1,2 & 27 & 18,8 & 19,8 & 1,0 & 24 \\
\hline Yucatán & 47,6 & 40,6 & $-7,0$ & 7 & 26,4 & 23,8 & $-2,6$ & 16 \\
\hline
\end{tabular}




\begin{tabular}{|c|c|c|c|c|c|c|c|c|}
\hline Veracruz & 41,0 & 40,7 & $-0,3$ & 24 & 17,6 & 12,5 & $-5,1$ & $\underline{6}$ \\
\hline \multicolumn{9}{|l|}{ BAJO } \\
\hline SLP & 47,3 & 39,5 & $-7,8$ & $\underline{\mathbf{3}}$ & 24,5 & 21,2 & $-3,3$ & 11 \\
\hline Hidalgo & 43,8 & 37,8 & $-6,0$ & 11 & 25,7 & 18,2 & $-7,5$ & $\underline{1}$ \\
\hline Nayarit & 50,8 & 45,8 & $-5,0$ & 12 & 24,1 & 27,3 & 3,2 & 28 \\
\hline Puebla & 49,9 & 42,0 & $-7,9$ & $\underline{2}$ & 26,6 & 19,4 & $-7,2$ & $\underline{2}$ \\
\hline Zacatecas & 55,4 & 48,0 & $-7,4$ & $\underline{5}$ & 26,4 & 26,1 & $-0,3$ & 22 \\
\hline Michoacán & 55,4 & 48,2 & $-7,2$ & $\underline{6}$ & 24,3 & 23,7 & $-0,6$ & 19 \\
\hline \multicolumn{9}{|l|}{ MUY BAJO } \\
\hline Chiapas & 55,3 & 55,8 & 0,5 & 26 & 14,2 & 16,4 & 2,2 & 26 \\
\hline Guerrero & 49,4 & 56,8 & 7,4 & 32 & 21,8 & 18,6 & $-3,2$ & 12 \\
\hline Oaxaca & 47,8 & 47,0 & $-0,8$ & 21 & 21,4 & 24,2 & 2,8 & 27 \\
\hline NACIONAL & 43,0 & 40,4 & $-2,6$ & & 21,6 & 19,7 & $-1,8$ & \\
\hline
\end{tabular}

'El grado de asalarización de la PEA total de la entidad federativa.

${ }^{2}$ Ordenamiento decreciente de las entidades federativas por las diferencias porcentuales entre 2008 y 2009 . El valor 1 corresponde a la diferencia más elevada. En negrillas y subrayado están las entidades federativas que ocupan los primeros diez lugares.

Fuente: Elaborados a partir de los datos de la ENOE, segundo semestres 2008-2009 
CUADRO 5

DIFERENCIAS PORCENTUALES DE TASAS ENTRE 2008-2009 DE DESEMPLEO, I NFORMALIDAD Y DESPROTECCIÓN DE LA PEA JÓVEN POR ENTIDAD FEDERATIVA' HOMBRES DE 14-19 AÑOS

\begin{tabular}{|c|c|c|c|c|c|c|}
\hline \multirow[b]{2}{*}{$\begin{array}{c}\text { Grado de } \\
\text { asalarización }^{2}\end{array}$} & \multicolumn{2}{|c|}{ Desempleo } & \multicolumn{2}{|c|}{ Informalidad } & \multicolumn{2}{|c|}{ Desprotección } \\
\hline & $\begin{array}{c}\text { Nivel de } \\
\text { incremento }\end{array}$ & $\begin{array}{c}\text { Rango de } \\
\text { incremento }\end{array}$ & $\begin{array}{c}\text { Nivel de } \\
\text { incremento }\end{array}$ & $\begin{array}{l}\text { Rango de } \\
\text { incremento }\end{array}$ & $\begin{array}{c}\text { Nivel de } \\
\text { incremento }\end{array}$ & $\begin{array}{c}\text { Rango de } \\
\text { incremento }\end{array}$ \\
\hline \multicolumn{7}{|l|}{ MUY ALTO } \\
\hline Nuevo León & 6,9 & $\underline{5}$ & $-1,8$ & 22 & 7,0 & $\underline{7}$ \\
\hline Aguascalientes & 6,8 & $\underline{7}$ & $-3,6$ & 29 & 3,6 & 14 \\
\hline Coahuila & 5,2 & 10 & $-9,1$ & 31 & 11,8 & $\underline{1}$ \\
\hline Baja California Sur & 1,3 & 27 & $-2,9$ & 25 & 0,1 & 21 \\
\hline Baja California & 6,9 & $\underline{6}$ & 4 & $\underline{8}$ & 5,4 & $\underline{10}$ \\
\hline \multicolumn{7}{|l|}{ ALTO } \\
\hline Distrito Federal & 3 & 18 & 6,8 & $\underline{1}$ & $-5,7$ & 29 \\
\hline Querétaro & 6,6 & $\underline{8}$ & 1,7 & 14 & $-3,3$ & 27 \\
\hline Tamaulipas & 10,5 & 1 & 3,7 & $\underline{9}$ & 0,8 & 19 \\
\hline México & 5,8 & $\underline{\mathbf{9}}$ & $-0,5$ & 20 & 0,1 & 22 \\
\hline Chihuahua & $-1,1$ & 31 & 4,9 & $\underline{5}$ & 7,0 & $\underline{6}$ \\
\hline Sonora & 8,9 & $\underline{2}$ & 5,1 & $\underline{4}$ & $-13,2$ & 31 \\
\hline Durango & 2,1 & 21 & 4,2 & 7 & 4,3 & 12 \\
\hline Quintana Roo & 8,7 & $\underline{3}$ & 6,5 & $\underline{2}$ & 6,7 & $\underline{8}$ \\
\hline Tabasco & 3,9 & 15 & 3,7 & 10 & $-1,8$ & 26 \\
\hline \multicolumn{7}{|l|}{ MODERADO } \\
\hline Jalisco & $-0,4$ & 29 & $-2,6$ & 24 & 0,6 & 20 \\
\hline Colima & 1,8 & 25 & 3,7 & 11 & 7,2 & 4 \\
\hline Sinaloa & 4,3 & 13 & 4,8 & $\underline{6}$ & $-10,2$ & 30 \\
\hline Tlaxcala & 1,9 & 22 & $-4,6$ & 30 & 6,6 & $\underline{9}$ \\
\hline Morelos & 2,7 & 20 & $-14,1$ & 32 & 11,7 & 2 \\
\hline Guanajuato & 5,1 & 11 & 6,3 & $\underline{3}$ & 0,0 & 23 \\
\hline Campeche & 3,1 & 17 & 1,9 & 13 & 3,7 & 13 \\
\hline Yucatán & 1,9 & 24 & 0,6 & 16 & $-1,0$ & 25 \\
\hline Veracruz & $-1,1$ & 32 & 0,1 & 18 & $-0,2$ & 24 \\
\hline
\end{tabular}




\begin{tabular}{|c|c|c|c|c|c|c|}
\hline \multicolumn{7}{|l|}{ BAJO } \\
\hline SLP & 3,8 & 16 & $-1,7$ & 21 & 7,1 & $\underline{5}$ \\
\hline Hidalgo & 4,3 & 14 & 2,1 & 12 & $-4,7$ & 28 \\
\hline Nayarit & 3 & 19 & $-2,1$ & 23 & 2,0 & 18 \\
\hline Puebla & 4,4 & 12 & 1,1 & 15 & 3,1 & 16 \\
\hline Zacatecas & 0,7 & 28 & -3 & 26 & 5,2 & 11 \\
\hline Michoacán & 7,1 & $\underline{4}$ & $-3,2$ & 27 & 2,4 & 17 \\
\hline \multicolumn{7}{|l|}{ MUY BAJO } \\
\hline Chiapas & $-0,7$ & 30 & 0,4 & 17 & 3,4 & 15 \\
\hline Guerrero & 1,6 & 26 & $-3,5$ & 28 & 9,1 & $\underline{3}$ \\
\hline Oaxaca & 1,9 & 23 & $-0,4$ & 19 & $-20,3$ & 32 \\
\hline
\end{tabular}

${ }^{1}$ Las definiciones de desempleo, informalidad y desprotección se encuentran en el texto.

${ }^{2}$ El grado de asalarización de la PEA total de la entidad federativa.

${ }^{3}$ Ordenamiento decreciente de las entidades federativas por las diferencias porcentuales entre 2008 y 2009 . El valor 1 corresponde al mayor incremento. En negrillas y subrayado están las entidades federativas que ocupan los primeros diez lugares.

Fuente: Elaborados a partir de los datos de la ENOE, segundo trimestre 2008-2009. 
CUADRO 6

DIFERENCIAS PORCENTUALES DE TASAS ENTRE 2008 - 2009 EN LOS

INDICADORES SELECCIONADOS POR ENTIDADES FEDERATIVAS

MUJERES DE 14-19 AÑOS

\begin{tabular}{|c|c|c|c|c|c|c|}
\hline \multirow[b]{2}{*}{ Grado de asalarización ${ }^{1}$} & \multicolumn{2}{|c|}{ Desempleo } & \multicolumn{2}{|c|}{ Informalidad } & \multicolumn{2}{|c|}{ Desprotección } \\
\hline & Diferencias & Rango $^{2}$ & Diferencias & Rango & Diferencia & Rango \\
\hline \multicolumn{7}{|l|}{ MUY ALTO } \\
\hline Nuevo León & 10,2 & $\underline{\mathbf{3}}$ & 1,6 & 19 & $-4,3$ & 23 \\
\hline Aguascalientes & 3,2 & 11 & $-7,2$ & 30 & 6,8 & $\underline{6}$ \\
\hline Coahuila & 0,5 & 22 & $-6,2$ & 29 & 12,9 & $\underline{\mathbf{2}}$ \\
\hline Baja California Sur & 11,3 & 1 & $-4,1$ & 28 & $-5,2$ & 25 \\
\hline Baja California & 9,3 & $\underline{4}$ & 5,2 & 11 & 4,8 & $\underline{8}$ \\
\hline \multicolumn{7}{|l|}{ ALTO } \\
\hline DF & 1,8 & 16 & 1,5 & 20 & 8,0 & $\underline{\mathbf{5}}$ \\
\hline Querétaro & 4 & $\underline{9}$ & $-3,0$ & 24 & 20,3 & 1 \\
\hline Tamaulipas & 3,3 & $\underline{10}$ & 3,3 & 16 & $-4,3$ & 24 \\
\hline México & 0,8 & 20 & $-0,3$ & 23 & 9,9 & $\underline{3}$ \\
\hline Chihuahua & 7,8 & $\underline{6}$ & $-8,3$ & 31 & 2,9 & 13 \\
\hline Sonora & 10,6 & $\underline{2}$ & 13,4 & 4 & $-0,8$ & 19 \\
\hline Durango & 9,3 & $\underline{5}$ & 24,4 & 1 & $-11,6$ & 31 \\
\hline Quintana Roo & 2,8 & 13 & 3,5 & 15 & 2,1 & 15 \\
\hline Tabasco & 2,7 & 14 & $-3,1$ & 25 & 9,1 & 4 \\
\hline \multicolumn{7}{|l|}{ MODERADO } \\
\hline Jalisco & 4,8 & $\underline{8}$ & 3,2 & 17 & $-3,7$ & 22 \\
\hline Colima & 3,2 & 12 & 10,6 & $\underline{5}$ & $-1,0$ & 20 \\
\hline Sinaloa & 0,1 & 26 & 17,4 & $\underline{2}$ & $-20,0$ & 32 \\
\hline Tlaxcala & $-2,3$ & 30 & 1,5 & 21 & 4,0 & $\underline{10}$ \\
\hline Morelos & $-3,9$ & 31 & 4,0 & 14 & $-1,3$ & 21 \\
\hline Guanajuato & 0,4 & 24 & 1,3 & 22 & 0,9 & 16 \\
\hline Campeche & 7,4 & $\underline{7}$ & 15,8 & $\underline{3}$ & $-8,4$ & 29 \\
\hline Yucatán & $-0,9$ & 27 & 9,8 & $\underline{6}$ & $-8,3$ & 28 \\
\hline Veracruz & 1,9 & 15 & 6,1 & $\underline{10}$ & 3,2 & 12 \\
\hline
\end{tabular}




\begin{tabular}{|c|c|c|c|c|c|c|}
\hline \multicolumn{7}{|l|}{ BAJO } \\
\hline SLP & 0,8 & 21 & 7,8 & $\underline{9}$ & $-5,5$ & 26 \\
\hline Hidalgo & -7 & 32 & 8,1 & $\underline{8}$ & 2,3 & 14 \\
\hline Nayarit & 1,4 & 17 & 4,4 & 12 & 4,5 & $\underline{9}$ \\
\hline Puebla & 0,4 & 25 & $-10,1$ & 32 & 6,8 & $\underline{7}$ \\
\hline Zacatecas & $-2,2$ & 29 & 4,2 & 13 & $-0,3$ & 17 \\
\hline Michoacán & 0,5 & 23 & 9,0 & $\underline{7}$ & $-0,4$ & 18 \\
\hline \multicolumn{7}{|l|}{ MUY BAJO } \\
\hline Chiapas & -1 & 28 & 2,2 & 18 & 3,5 & 11 \\
\hline Guerrero & 1 & 18 & $-3,5$ & 27 & $-6,4$ & 27 \\
\hline Oaxaca & 1 & 19 & $-3,2$ & 26 & $-9,7$ & 30 \\
\hline
\end{tabular}

`El grado de asalarización de la PEA total de la entidad federativa.

2Ordenamiento decreciente de las entidades federativas por las diferencias porcentuales entre 2008 y 2009.

El valor 1 corresponde al mayor incremento. En negrillas y subrayado están las entidades federativas que ocupan los primeros diez lugares.

Fuente: Elaborados a partir de los datos de la ENOE, segundo trimestre 2008-2009 
CUADRO 7

TASAS DE DESEMPLEO, INFORMALIDAD Y DESPROTECCIÓN DE LA PEA JÓVEN POR ENTIDADES FEDERATIVAS, 2008-2009 HOMBRES DE 14-19 AÑOS.

\begin{tabular}{|c|c|c|c|c|c|c|}
\hline \multirow{2}{*}{$\begin{array}{c}\text { Grado de } \\
\text { asalarización }{ }^{1}\end{array}$} & \multicolumn{2}{|c|}{ Desempleo } & \multicolumn{2}{|c|}{ Informalidad } & \multicolumn{2}{|c|}{ Desprotección ${ }^{2}$} \\
\hline & 2008 & 2009 & 2008 & 2009 & 2008 & 2009 \\
\hline \multicolumn{7}{|l|}{ MUY ALTO } \\
\hline Nuevo León & 12,4 & 19,3 & 26,9 & 25,1 & 21,5 & 28,5 \\
\hline Aguascalientes & 9,4 & 16,2 & 40,9 & 37,3 & 34,9 & 38,5 \\
\hline Coahuila & 14,2 & 19,4 & 37,3 & 28,2 & 25,4 & 37,2 \\
\hline Baja California Sur & 8,8 & 10,1 & 27,1 & 24,2 & 32,9 & 33,0 \\
\hline Baja California & 7,6 & 14,5 & 17,8 & 21,8 & 32,0 & 37,4 \\
\hline \multicolumn{7}{|l|}{ ALTO } \\
\hline Distrito Federal & 9,2 & 12,2 & 41,5 & 48,3 & 36,4 & 30,7 \\
\hline Querétaro & 9,8 & 16,4 & 30,4 & 32,1 & 44,5 & 41,2 \\
\hline Tamaulipas & 5,9 & 16,4 & 25,4 & 29,1 & 36,0 & 36,8 \\
\hline México & 9,1 & 14,9 & 49,3 & 48,8 & 28,8 & 28,9 \\
\hline Chihuahua & 14,7 & 13,6 & 18,1 & 23,0 & 37,0 & 44,0 \\
\hline Sonora & 6,7 & 15,6 & 29,5 & 34,6 & 41,7 & 28,5 \\
\hline Durango & 7,9 & 10,0 & 31,6 & 35,8 & 40,1 & 44,4 \\
\hline Quintana Roo & 4,4 & 13,1 & 26,3 & 32,8 & 37,3 & 44,0 \\
\hline Tabasco & 3,8 & 7,7 & 29,4 & 33,1 & 48,8 & 47,0 \\
\hline \multicolumn{7}{|l|}{ MODERADO } \\
\hline Jalisco & 8,5 & 8,1 & 34,7 & 32,1 & 41,7 & 42,3 \\
\hline Colima & 4,4 & 6,2 & 22,3 & 26,0 & 42,6 & 49,8 \\
\hline Sinaloa & 3,6 & 7,9 & 20,7 & 25,5 & 49,9 & 39,7 \\
\hline Tlaxcala & 9,1 & 11,0 & 51,2 & 46,6 & 33,5 & 40,1 \\
\hline Morelos & 3,8 & 6,5 & 46,3 & 32,2 & 37,3 & 49,0 \\
\hline Guanajuato & 6,2 & 11,3 & 29,9 & 36,2 & 47,9 & 47,9 \\
\hline Campeche & 1,5 & 4,6 & 20,6 & 22,5 & 51,6 & 55,3 \\
\hline Yucatán & 4,9 & 6,8 & 32,4 & 33,0 & 48,8 & 47,8 \\
\hline Veracruz & 4,6 & 3,5 & 26,4 & 26,5 & 58,3 & 58,1 \\
\hline
\end{tabular}




\begin{tabular}{|c|c|c|c|c|c|c|}
\hline \multicolumn{7}{|l|}{ BAJO } \\
\hline San Luis Potosí & 4,5 & 8,3 & 29,4 & 27,7 & 39,5 & 46,6 \\
\hline Hidalgo & 3,5 & 7,8 & 34,4 & 36,5 & 52,0 & 47,3 \\
\hline Nayarit & 5,9 & 8,9 & 28,9 & 26,8 & 43,7 & 45,7 \\
\hline Puebla & 2,2 & 6,6 & 36,5 & 37,6 & 47,4 & 50,5 \\
\hline Zacatecas & 7,9 & 8,6 & 25,1 & 22,1 & 54,0 & 59,2 \\
\hline Michoacán & 3,4 & 10,5 & 39,6 & 36,4 & 44,3 & 46,7 \\
\hline \multicolumn{7}{|l|}{ MUY BAJO } \\
\hline Chiapas & 2,7 & 2,0 & 15,2 & 15,6 & 54,1 & 57,5 \\
\hline Guerrero & 0,3 & 1,7 & 27,7 & 24,2 & 58,1 & 67,2 \\
\hline Oaxaca & 1,8 & 3,7 & 35,6 & 35,2 & 55,2 & 34,9 \\
\hline NACIONAL & 6,3 & 9,7 & 32,4 & 32,5 & 42,9 & 44,2 \\
\hline
\end{tabular}

El grado de asalarización de la PEA total de la entidad federativa.

2trabajadores sin seguro social. No incluye a los trabajadores en el sector informal.

Fuente: Elaborados a partir de los datos de la ENOE, segundo trimestre 2008-2009. 
CUADRO 8

TASAS DE DESEMPLEO, INFORMALIDAD, Y DESPROTECCIÓN CLASIFICADAS

POR GRADO DE ASALARIZACIÓN DE LAS ENTIDADES FEDERATIVAS 2008-2009

MUJERES DE 14-19 AÑOS

\begin{tabular}{|c|c|c|c|c|c|c|}
\hline \multirow{2}{*}{$\begin{array}{c}\text { Grado de } \\
\text { asalarización }{ }^{1}\end{array}$} & \multicolumn{2}{|c|}{ Desempleo } & \multicolumn{2}{|c|}{ Informalidad } & \multicolumn{2}{|c|}{ Desprotección ${ }^{2}$} \\
\hline & 2008 & 2009 & 2008 & 2009 & 2008 & 2009 \\
\hline \multicolumn{7}{|l|}{ MUY ALTO } \\
\hline Nuevo León & 14,8 & 25,0 & 19,9 & 21,5 & 33,3 & 29 \\
\hline Aguascalientes & 7,4 & 10,6 & 28,2 & 21 & 43,5 & 50,3 \\
\hline Coahuila & 16,9 & 17,4 & 34,9 & 28,7 & 24,3 & 37,2 \\
\hline Baja California Sur & 3,6 & 14,9 & 14,3 & 10,2 & 40,3 & 35,1 \\
\hline Baja California & 7,2 & 16,5 & 12,2 & 17,4 & 37,6 & 42,4 \\
\hline \multicolumn{7}{|l|}{ ALTO } \\
\hline Distrito Federal & 13,6 & 15,4 & 33,3 & 34,8 & 37,3 & 45,3 \\
\hline Querétaro & 5,6 & 9,6 & 17,2 & 14,2 & 38,4 & 58,7 \\
\hline Tamaulipas & 19,5 & 22,8 & 21,3 & 24,6 & 37,3 & 33 \\
\hline México & 12,5 & 13,3 & 36,7 & 36,4 & 32,8 & 42,7 \\
\hline Chihuahua & 5,4 & 13,2 & 11,1 & 2,8 & 21,2 & 24,1 \\
\hline Sonora & 8,7 & 19,3 & 23,3 & 36,7 & 33,9 & 33,1 \\
\hline Durango & 9,4 & 18,7 & 26,9 & 50,5 & 41,7 & 30,1 \\
\hline Quintana Roo & 10,4 & 13,2 & 24,4 & 27,9 & 39,7 & 41,8 \\
\hline Tabasco & 8,7 & 11,4 & 31,2 & 28,1 & 49,2 & 58,3 \\
\hline \multicolumn{7}{|l|}{ MODERADO } \\
\hline Jalisco & 6,1 & 10,9 & 27,7 & 30,9 & 40,6 & 36,9 \\
\hline Colima & 5,0 & 8,2 & 14,8 & 25,4 & 54 & 53 \\
\hline Sinaloa & 5,8 & 5,9 & 17,2 & 34,6 & 50,2 & 30,2 \\
\hline Tlaxcala & 12,1 & 9,8 & 47,2 & 48,7 & 36 & 40 \\
\hline Morelos & 6,1 & 2,9 & 32,2 & 36,2 & 47,3 & 46 \\
\hline Guanajuato & 6,3 & 6,7 & 31,5 & 32,8 & 45,7 & 46,6 \\
\hline Campeche & 1,9 & 9,3 & 29,4 & 45,2 & 46,6 & 38,2 \\
\hline Yucatán & 4,3 & 3,7 & 37,8 & 47,6 & 46,1 & 37,8 \\
\hline Veracruz & 2,4 & 4,3 & 27,4 & 33,5 & 51,8 & 55 \\
\hline
\end{tabular}




\begin{tabular}{|l|c|c|c|c|c|c|}
\hline \multicolumn{7}{|l|}{ BAJO } \\
\hline San Luis Potosí & 7,6 & 8,4 & 24,1 & 31,9 & 51,8 & 46,3 \\
\hline Hidalgo & 9,9 & 2,9 & 25,9 & 34 & 49,3 & 51,6 \\
\hline Nayarit & 3,0 & 4,4 & 35,9 & 40,3 & 33,9 & 38,4 \\
\hline Puebla & 6,5 & 6,9 & 45,7 & 35,6 & 38,5 & 45,3 \\
\hline Zacatecas & 10,6 & 8,4 & 23,4 & 27,6 & 50,4 & 50,1 \\
\hline Michoacán & 9,1 & 9,6 & 31,8 & 40,8 & 46,7 & 46,3 \\
\hline MuY BAJO & & & & & & \\
\hline Chiapas & 2,4 & 1,0 & 29,6 & 31,8 & 50,6 & 54,1 \\
\hline Guerrero & 1,2 & 1,3 & 46,8 & 43,3 & 45,78 & 39,4 \\
\hline Oaxaca & 1,9 & 2,9 & 52,7 & 49,5 & 41,7 & 32,0 \\
\hline NACIONAL & $\mathbf{8 , 2}$ & $\mathbf{1 0 , 4}$ & $\mathbf{3 0 , 9}$ & $\mathbf{3 3 , 5}$ & $\mathbf{4 1 , 2}$ & $\mathbf{4 2 , 2}$ \\
\hline
\end{tabular}

'El grado de asalarización de la PEA total de la entidad federativa.

${ }^{2}$ trabajadores sin seguro social. No incluye a los trabajadores en el sector informal.

Fuente: Elaborados a partir de los datos de la ENOE, segundo trimestre 2008-2009. 
CUADRO 9

DIFERENCIAS PORCENTUALES EN LAS TASAS DE DESEMPLEO, INFORMALIDAD

Y DESPROTECCIÓN DE LA PEA TOTAL, ENTRE 2008-2009

\begin{tabular}{|c|c|c|c|c|c|c|}
\hline \multirow[b]{2}{*}{ Grado de asalarización' } & \multicolumn{2}{|c|}{ Desempleo } & \multicolumn{2}{|c|}{ Informalidad } & \multicolumn{2}{|c|}{ Desprotección ${ }^{2}$} \\
\hline & Diferencia & Rango $^{3}$ & Diferencia & Rango & Diferencia & Rango \\
\hline \multicolumn{7}{|l|}{ MUY ALTO } \\
\hline Nuevo León & 2,9 & $\underline{5}$ & $-1,2$ & 30 & 0,2 & 18 \\
\hline Aguascalientes & 2,3 & $\underline{9}$ & $-1,1$ & 29 & 1,1 & 12 \\
\hline Coahuila & 2,9 & 4 & $-0,6$ & 25 & 0,8 & 13 \\
\hline Baja California Sur & 2,3 & 8 & 1,8 & $\underline{\mathbf{5}}$ & $-0,3$ & 23 \\
\hline Baja California & 3,0 & $\underline{3}$ & 1,8 & $\underline{6}$ & 2,6 & $\underline{4}$ \\
\hline \multicolumn{7}{|l|}{ ALTO } \\
\hline Distrito Federal & 1,4 & 17 & 0,6 & 19 & $-0,0$ & 20 \\
\hline Querétaro & 4,5 & $\underline{1}$ & 0,2 & 21 & 1,8 & $\underline{7}$ \\
\hline Tamaulipas & 2,0 & 12 & 1,5 & 12 & 1,4 & $\underline{9}$ \\
\hline México & 2,8 & 6 & $-0,4$ & 23 & 1,3 & $\underline{10}$ \\
\hline Chihuahua & 3,1 & $\underline{2}$ & 2,5 & $\underline{3}$ & 3,6 & $\underline{2}$ \\
\hline Sonora & 1,6 & 13 & 2,9 & $\underline{2}$ & $-2,0$ & 31 \\
\hline Durango & 1,6 & 15 & 4,6 & 1 & $-0,8$ & 28 \\
\hline Quintana Roo & 2,6 & $\underline{7}$ & $-1,1$ & 28 & 2,1 & $\underline{5}$ \\
\hline Tabasco & 1,6 & 14 & 0,8 & 16 & 1,2 & 11 \\
\hline \multicolumn{7}{|l|}{ MODERADO } \\
\hline Jalisco & 1,4 & 16 & 0,7 & 17 & $-0,2$ & 21 \\
\hline Colima & 2,0 & 11 & 1,6 & $\underline{9}$ & 1,5 & $\underline{8}$ \\
\hline Sinaloa & 1,3 & 20 & 0,9 & 15 & $-1,9$ & 30 \\
\hline Tlaxcala & 0,9 & 24 & $-0,8$ & 27 & 4,6 & 1 \\
\hline Morelos & 0,9 & 25 & $-3,8$ & 32 & 0,5 & 15 \\
\hline Guanajuato & 1,1 & 21 & 1,0 & 14 & 0,6 & 14 \\
\hline Campeche & 0,8 & 26 & 1,8 & $\underline{7}$ & $-0,0$ & 19 \\
\hline Yucatán & 0,5 & 28 & 1,5 & 11 & $-0,3$ & 24 \\
\hline Veracruz & 0,7 & 27 & 1,6 & $\underline{8}$ & $-0,8$ & 27 \\
\hline
\end{tabular}




\begin{tabular}{|c|c|c|c|c|c|c|}
\hline \multicolumn{7}{|l|}{ BAJO } \\
\hline San Luis Potosí & 0,9 & 23 & 0,5 & 20 & $-0,5$ & 26 \\
\hline Hidalgo & 2,0 & $\underline{10}$ & 0,1 & 22 & 0,2 & 17 \\
\hline Nayarit & 0,9 & 22 & $-0,8$ & 26 & 1,9 & $\underline{6}$ \\
\hline Puebla & 1,4 & 18 & 1,6 & $\underline{10}$ & $-0,4$ & 25 \\
\hline Zacatecas & 0,1 & 31 & 2,3 & $\underline{4}$ & $-0,2$ & 22 \\
\hline Michoacán & 1,3 & 19 & 0,7 & 18 & 0,4 & 16 \\
\hline \multicolumn{7}{|l|}{ MUY BAJO } \\
\hline Chiapas & 0,0 & 32 & 1,2 & 13 & $-1,2$ & 29 \\
\hline Guerrero & 0,4 & 29 & $-0,6$ & 24 & 2,7 & $\underline{3}$ \\
\hline Oaxaca & 0,3 & 30 & $-1,5$ & 31 & $-3,0$ & 32 \\
\hline
\end{tabular}

`El grado de asalarización de la PEA total de la entidad federativa.

${ }^{2}$ trabajadores sin seguro social. No incluye a los trabajadores en el sector informal.

${ }^{3}$ Ordenamiento decreciente de las entidades federativas por las diferencias porcentuales entre 2008 y 2009. El valor 1 corresponde al mayor incremento. En negrillas y subrayado están las entidades federativas que ocupan los primeros diez lugares.

Fuente: Elaborados a partir de los datos de la ENOE, segundo trimestre 2008-2009. 
CUADRO 10

DISTRIBUCIÓN DEL PIB DE LAS ENTIDADES FEDERATIVAS SELECCIONADAS

SEGÚN SECTORES DE ACTIVIDAD, 2008 A PRECIOS DE 2003

INDUSTRIAS MANUFACTURERAS

\begin{tabular}{|c|c|c|c|c|c|c|c|}
\hline \multirow[b]{2}{*}{ Grado de asalarización ${ }^{1}$} & \multirow[b]{2}{*}{ Agricultura } & \multirow[b]{2}{*}{ Minería } & \multicolumn{3}{|c|}{ Industria } & \multirow[b]{2}{*}{$\begin{array}{c}\text { Construc- } \\
\text { ción }\end{array}$} & \multirow[b]{2}{*}{$\begin{array}{l}\text { Actividades } \\
\text { terciarias }\end{array}$} \\
\hline & & & $\begin{array}{c}\text { Total } \\
\text { industria }\end{array}$ & $\begin{array}{c}\text { Maquinaria } \\
\text { y equipo }\end{array}$ & $\begin{array}{l}\text { Industria } \\
\text { Alimentaria }\end{array}$ & & \\
\hline \multicolumn{8}{|l|}{ MUY ALTO } \\
\hline Nuevo leon & 0,7 & 1,4 & $\underline{24,5}$ & $\underline{7,9}$ & 4,4 & 9,4 & 62,6 \\
\hline Coahuila & 2,8 & 2,9 & $\underline{35,3}$ & $\underline{17,5}$ & 3,4 & 7,1 & 50,4 \\
\hline Baja california sur & 5,5 & 2,7 & 3,2 & 0,0 & 2,4 & $\underline{11,5}$ & 75,1 \\
\hline Baja californa & 3,0 & 0,2 & $\underline{22,0}$ & $\underline{10,0}$ & 3,9 & 9,5 & 63,0 \\
\hline \multicolumn{8}{|l|}{ ALTO } \\
\hline Queretaro & 2,8 & 0,9 & $\underline{24,5}$ & 10,6 & 4,9 & 10,0 & 60,9 \\
\hline Chihuahua & 5,8 & 0,6 & $\underline{25,8}$ & $\underline{14,9}$ & 3,2 & 6,9 & 59,4 \\
\hline Sonora & $\underline{8,5}$ & 2,0 & $\underline{20,2}$ & $\underline{8,3}$ & 6,2 & 7,4 & 60,4 \\
\hline Durango & $\underline{12,3}$ & 2,1 & $\underline{21,3}$ & 2,2 & $\underline{12,2}$ & 6,3 & 56,2 \\
\hline DF & 0,1 & 0,0 & 10,6 & 1,0 & 2,5 & 3,8 & 85,1 \\
\hline Quintana Roo & 0,6 & 0,3 & 2,3 & 0,0 & 1,4 & $\underline{10,3}$ & 85,7 \\
\hline Tamaulipas & 3,5 & $\underline{7,4}$ & 13,3 & 5,3 & 2,2 & 16,1 & 56,9 \\
\hline \multicolumn{8}{|l|}{ MODERADO } \\
\hline Guanajuato & 4,6 & 0,3 & $\underline{27,6}$ & $\underline{9,1}$ & 8,1 & 6,8 & 59,5 \\
\hline Sinaloa & $\underline{13,0}$ & 0,4 & 8,2 & 0,6 & 6,2 & 7,0 & 69,5 \\
\hline Campeche & 0,8 & $\underline{76,3}$ & 0,7 & 0,0 & 0,4 & 6,9 & 15,1 \\
\hline \multicolumn{8}{|l|}{ BAJO } \\
\hline Michoacan & $\underline{10,6}$ & 0,4 & 13,5 & 0,2 & 4,3 & 5,9 & 68,0 \\
\hline San Luis Potosí & 4,5 & 1,2 & $\underline{25,6}$ & 6,6 & 7,5 & 6,9 & 58,7 \\
\hline Puebla & 4,4 & 0,8 & $\underline{29,7}$ & $\underline{15,7}$ & 6,0 & 5,5 & 52,4 \\
\hline Hidalgo & 5,0 & 1,2 & $\underline{28,2}$ & 2,6 & 8,8 & $\underline{10,9}$ & 52,4 \\
\hline \multicolumn{8}{|l|}{ MUY BAJO } \\
\hline Guerrero & $\underline{7,0}$ & 0,7 & 6,2 & 0,0 & 3,8 & 6,0 & 77,4 \\
\hline Oaxaca & $\underline{9,9}$ & 0,1 & 14,7 & 0,0 & 5,6 & 4,2 & 70,0 \\
\hline
\end{tabular}

'El grado de asalarización de la PEA total de la entidad federativa.

Fuente: Elaborado a partir de los datos nacionales, INEGI. 
CUADRO 11

DISTRIBUCIÓN DEL PIB DE LAS ENTIDADES FEDERATIVAS SELECCIONADAS

SEGÚN LAS PRINCIPALES RAMAS DE ACTIVIDAD DEL SECTOR TERCIARIO 2008,

A PRECIOS DE 2003'

\begin{tabular}{|c|c|c|c|c|c|c|c|c|c|c|}
\hline 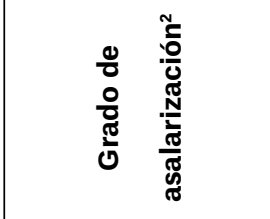 & $\begin{array}{l}\frac{0}{0} \\
\frac{0}{d} \\
\dot{0} \\
0\end{array}$ & 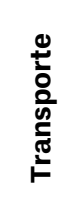 & 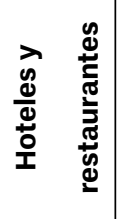 & 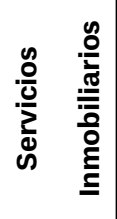 & 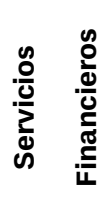 & 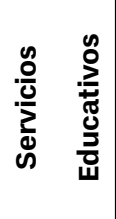 & 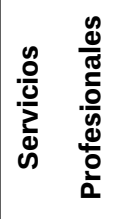 & 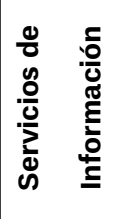 & 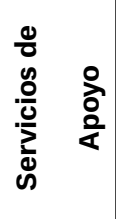 & 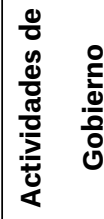 \\
\hline \multicolumn{11}{|l|}{ MUY ALTA } \\
\hline Nuevo leon & 16,5 & 8,5 & 1,6 & 9,6 & 6,8 & 3,6 & 4,8 & 4,1 & 4,0 & 1,7 \\
\hline Coahuila & 13,1 & 8,0 & 2,0 & 7,9 & 2,0 & 3,5 & 3,3 & 1,9 & 2,3 & 2,6 \\
\hline Baja California Sur & $\underline{18,4}$ & 8,9 & $\underline{13,9}$ & 9,5 & 4,0 & 4,0 & 2,1 & 3,0 & 2,4 & 5,3 \\
\hline Baja California & 17,1 & 7,3 & 3,2 & 11,6 & 2,2 & 4,4 & 3,4 & 3,8 & 1,4 & 3,9 \\
\hline \multicolumn{11}{|l|}{ ALTA } \\
\hline Queretaro & $\underline{17,7}$ & $\underline{10,9}$ & 2,1 & 8,1 & 1,8 & 4,1 & 3,2 & 4,7 & 1,8 & 2,8 \\
\hline Chihuhua & 15,8 & 4,7 & 2,9 & 11,5 & 2,0 & 4,4 & 6,0 & 3,3 & 1,3 & 3,0 \\
\hline Sonora & 16,9 & 6,6 & 2,9 & 10,7 & 2,7 & 4,5 & 2,1 & 3,9 & 2,3 & 3,5 \\
\hline Durango & 14,8 & 7,6 & 1,7 & 11,5 & 2,5 & 4,3 & 1,2 & 2,4 & 2,2 & 5,1 \\
\hline Distrito Federal & $\underline{18,4}$ & 8,9 & 2,2 & 9,1 & $\underline{14,6}$ & 4,6 & $\underline{8,4}$ & $\underline{7,3}$ & $\underline{5,5}$ & $\underline{6,5}$ \\
\hline Quintana Roo & 17,9 & 8,4 & $\underline{20,4}$ & 9,1 & 3,5 & 2,8 & 3,5 & 2,1 & $\underline{5,7}$ & 3,3 \\
\hline Tamaulipas & 15,1 & 8,9 & 3,0 & 11,6 & 1,6 & 3,4 & 2,1 & 2,5 & 1,3 & 3,0 \\
\hline \multicolumn{11}{|l|}{ MODERADA } \\
\hline Guanajuato & 16,2 & 7,8 & 2,0 & 11,2 & 2,5 & 3,8 & 4,6 & 2,3 & 1,5 & 2,7 \\
\hline Sinaloa & $\underline{21,6}$ & 7,8 & 4,4 & 12,0 & 2,7 & 5,0 & 2,1 & 2,8 & 1,2 & 3,6 \\
\hline Campeche & 2,8 & 2,2 & 0,7 & 2,3 & 0,4 & 0,9 & 1,7 & 0,6 & 1,5 & 0,9 \\
\hline \multicolumn{11}{|l|}{ BAJA } \\
\hline Michoacan & $\underline{18,4}$ & 7,5 & 2,5 & 13,3 & 1,8 & 6,5 & 1,6 & 3,0 & 2,5 & 4,1 \\
\hline San Luis Potosí & 15,2 & 6,3 & 2,7 & 13,3 & 2,0 & 5,2 & 1,7 & 2,2 & 1,5 & 4,2 \\
\hline Hidalgo & 10,8 & 7,8 & 1,1 & 13,6 & 1,6 & 6,0 & 0,8 & 2,2 & 1,0 & 4,0 \\
\hline \multicolumn{11}{|l|}{ MUY BAJA } \\
\hline Guerrero & 16,1 & $\underline{10,8}$ & 8,1 & $\underline{18,1}$ & 1,3 & $\underline{7,2}$ & 0,8 & 3,1 & 1,2 & 5,9 \\
\hline Oaxaca & 17,1 & 6,0 & 3,3 & $\underline{18,8}$ & 1,1 & $\underline{8,1}$ & 1,2 & 2,3 & 1,0 & 6,2 \\
\hline
\end{tabular}

'No se consideran los datos de Servicios de Intermediación Financiera Medidos Indirectamente (SIFMI)

de los datos nacionales, INEGI.

²El grado de asalarización de la PEA total de la entidad federativa.

Fuente: Elaborado a partir de los datos nacionales, INEGI. 
CUADRO 12

DISTRIBUCIÓN DE LA POBLACIÓN JOVEN OCUPADA POR RAMAS

DE ACTIVIDAD Y ENTIDADES FEDERATIVAS SELECCIONADAS

HOMBRES (14-19 AÑOS), 2008

\begin{tabular}{|c|c|c|c|c|c|c|}
\hline $\begin{array}{c}\text { Grado de } \\
\text { asalarización }{ }^{1}\end{array}$ & Agricultura & Construcción & Industria & Comercio & Servicios & Total \\
\hline \multicolumn{7}{|l|}{ MUY ALTA } \\
\hline Nvo. Leon & 3,4 & 13,4 & 28,5 & 19,8 & 34,9 & 100,0 \\
\hline Coahuila & 9,9 & 15,5 & 20,3 & 23,7 & 30,6 & 100,0 \\
\hline BCS & 15,6 & 21,6 & 10,9 & 23,8 & 28,1 & 100,0 \\
\hline BC & 18,7 & 13,0 & 16,2 & 15,5 & 36,6 & 100,0 \\
\hline \multicolumn{7}{|l|}{ ALTA } \\
\hline DF & 1,0 & 9,3 & 8,4 & 26,9 & 54,4 & 100,0 \\
\hline Queretaro & 37,0 & 10,1 & 17,2 & 15,4 & 20,2 & 100,0 \\
\hline Tamaulipas & 12,8 & 10,5 & 21,4 & 18,6 & 36,8 & 100,0 \\
\hline México & 11,2 & 13,8 & 19,1 & 21,5 & 34,3 & 100,0 \\
\hline Chihuahua & 18,3 & 13,5 & 16,6 & 15,7 & 36,0 & 100,0 \\
\hline Quintana Roo & 13,4 & 14,3 & 8,4 & 19,2 & 44,6 & 100,0 \\
\hline Durango & 31,1 & 15,6 & 19,3 & 14,3 & 19,7 & 100,0 \\
\hline \multicolumn{7}{|l|}{ MODERADA } \\
\hline Sinaloa & 11,2 & 13,8 & 19,2 & 21,5 & 34,3 & 100,0 \\
\hline Morelos & 20,6 & 21,3 & 10,8 & 27,7 & 19,5 & 100,0 \\
\hline Guanajuato & 23,8 & 11,9 & 23,4 & 15,9 & 25,1 & 100,0 \\
\hline \multicolumn{7}{|l|}{ BAJA } \\
\hline Michoacan & 41,0 & 15,6 & 14,6 & 17,1 & 11,7 & 100,0 \\
\hline \multicolumn{7}{|l|}{ MUY BAJA } \\
\hline Guerrero & 50,5 & 11,4 & 9,5 & 12,2 & 16,3 & 100,0 \\
\hline
\end{tabular}

${ }^{1}$ El grado de asalarización de la PEA total de la entidad federativa.

Fuente: Elaborado con base en la ENOE, segundo trimestre de 2008 
CUADRO 13

DISTRIBUCIÓN DE LA POBLACIÓN JOVEN OCUPADA POR RAMA DE ACTIVIDAD Y ENTIDADES FEDERATIVAS SELECCIONADAS,

MUJERES (14-19 AÑOS), 2008

\begin{tabular}{|c|c|c|c|c|c|c|}
\hline $\begin{array}{c}\text { Grado de } \\
\text { asalarización }{ }^{1}\end{array}$ & Agricultura & Construcción & Industria & Comercio & Servicios & Total \\
\hline \multicolumn{7}{|l|}{ MUY ALTO } \\
\hline Nuevo León & 0,2 & 0,0 & 23,0 & 26,7 & 50,1 & 100,0 \\
\hline Coahuila & 2,0 & 0,0 & 16,2 & 36,0 & 45,9 & 100,0 \\
\hline Baja California Sur & 14,3 & 5,7 & 2,0 & 30,2 & 47,8 & 100,0 \\
\hline Baja California & 4,1 & 0,7 & 16,8 & 26,8 & 51,6 & 100,0 \\
\hline \multicolumn{7}{|l|}{ ALTO } \\
\hline DF & 0,0 & 0,0 & 12,2 & 28,7 & 59,1 & 100,0 \\
\hline Queretaro & 12,0 & 0,2 & 29,2 & 23,7 & 34,9 & 100,0 \\
\hline Tamaulipas & 2,5 & 0,0 & 21,9 & 27,2 & 48,4 & 100,0 \\
\hline México & 2,6 & 0,1 & 23,4 & 24,6 & 49,3 & 100,0 \\
\hline Chihuahua & 1,8 & 0,0 & 39,8 & 16,0 & 42,5 & 100,0 \\
\hline Quintana Roo & 2,8 & 1,6 & 9,4 & 39,0 & 47,1 & 100,0 \\
\hline Durango & 2,5 & 0,0 & 18,4 & 34,6 & 44,5 & 100,0 \\
\hline \multicolumn{7}{|l|}{ MODERADO } \\
\hline Sinaloa & 2,6 & 0,1 & 23,4 & 24,6 & 49,3 & 100,0 \\
\hline Morelos & 1,2 & 1,4 & 17,5 & 31,7 & 48,1 & 100,0 \\
\hline Guanajuato & 8,0 & 0,0 & 36,8 & 33,1 & 22,1 & 100,0 \\
\hline \multicolumn{7}{|l|}{ BAJO } \\
\hline Michoacan & 9,0 & 0,0 & 17,2 & 36,9 & 36,9 & 100,0 \\
\hline \multicolumn{7}{|l|}{ MUY BAJO } \\
\hline Guerrero & 12,9 & 0,2 & 18,0 & 32,5 & 36,4 & 100,0 \\
\hline
\end{tabular}

'El grado de asalarización de la PEA total de la entidad federativa.

Fuente: Elaborados con base en la ENOE, segundo trimestre de 2008 